\title{
Homotopy Analysis Solution of Hydromagnetic Mixed Convection Flow Past an Exponentially Stretching Sheet with Hall Current
}

\author{
Mohamed Abd El-Aziz ${ }^{1,2}$ and Tamer Nabil ${ }^{1,3}$ \\ ${ }^{1}$ Mathematics Department, Faculty of Science, King Khalid University, Abha, P.O. Box 9004, Saudi Arabia \\ ${ }^{2}$ Mathematics Department, Faculty of Science, Helwan University, P.O. Box 11795, Cairo, Egypt \\ ${ }^{3}$ Basic Science Department, Faculty of Computers and Informatics, Suez Canal University, Ismailia, Egypt
}

Correspondence should be addressed to Mohamed Abd El-Aziz, m_abdelaziz999@yahoo.com

Received 9 May 2012; Revised 18 July 2012; Accepted 11 September 2012

Academic Editor: Claude Lamarque

Copyright (C) 2012 M. Abd El-Aziz and T. Nabil. This is an open access article distributed under the Creative Commons Attribution License, which permits unrestricted use, distribution, and reproduction in any medium, provided the original work is properly cited.

The effect of thermal radiation on steady hydromagnetic heat transfer by mixed convection flow of a viscous incompressible and electrically conducting fluid past an exponentially stretching continuous sheet is examined. Wall temperature and stretching velocity are assumed to vary according to specific exponential forms. An external strong uniform magnetic field is applied perpendicular to the sheet and the Hall effect is taken into consideration. The resulting governing equations are transformed into a system of nonlinear ordinary differential equations using appropriate transformations and then solved analytically by the homotopy analysis method (HAM). The solution is found to be dependent on six governing parameters including the magnetic field parameter $M$, Hall parameter $m$, the buoyancy parameter $\xi$, the radiation parameter $R$, the parameter of temperature distribution $a$, and Prandtl number Pr. A systematic study is carried out to illustrate the effects of these major parameters on the velocity and temperature distributions in the boundary layer, the skin-friction coefficients, and the local Nusselt number.

\section{Introduction}

In many industrial manufacturing processes, the problem of flow and heat transfer in twodimensional boundary layer on a continuous stretching surface, moving in an otherwise quiescent fluid medium, has attracted considerable attention during the last few decades. Examples may be found in continuous casting, glass-fiber production, hot rolling, wire drawing, paper production, drawing of plastic films, metal and polymer extrusion, and metal spinning. In recent years MHD flow problems have become more important in industry, since many metallurgical processes involve the cooling of continuous strips or filaments. By drawing them in an electrically conducting fluid in the presence of a magnetic field, 
the rate of cooling can be controlled. Another important application of hydromagnetics to metallurgy is the purification of molten metals from nonmetallic inclusions by the application of a magnetic field. The flow past a moving or stretching surface in an ambient fluid differs from that of the classical Blasius problem of flow past a stationary surface. The moving surface sucks the fluid and pumps it back in the downstream direction. Consequently, both the surface shear stress and the heat transfer are significantly enhanced. Sakiadis [1] firstly studied the boundary layer flow over a stretched surface moving with a constant velocity. Crane [2] extended this concept to a stretching sheet with linearly varying surface speed and presented an exact analytical solution for the steady two-dimensional stretching of a surface in a quiescent fluid. This problem has later been extensively studied in various directions including porous surface, non-Newtonian fluids, magnetohydrodynamic fluid, heat transfer, mass transfer, porous medium, and slip effects. Some interesting investigations are mentioned in the references [3-9]. It is known that the buoyancy force can produce significant changes in the velocity and temperature distribution and, hence, in the heat transfer rate from the surface. The effect of buoyancy force over continuous moving surfaces through an otherwise quiescent fluid was investigated by Chen and Strobel [10] and Fan et al. [11] for horizontal surfaces, by Chen [12], Ali and Al-Yousef [13, 14] and Abd El-Aziz and Salem [15] for vertical surfaces and by Moutsoglou and Chen [16], Strobel and Chen [17], and Chen [18] for vertical and inclined surfaces. A new dimension is added to the study of flow and heat transfer in a viscous fluid over a stretching surface by considering the effect of thermal radiation. We know that the radiation effect is important under many nonisothermal situations. If the entire system involving the polymer extrusion process is placed in a thermally controlled environment, then radiation could become important. Radiative heat transfer flow is very important in manufacturing industries for the design of reliable equipment, nuclear plants, gas turbines, and various propulsion devices for aircraft, missiles, satellites, and space vehicles. Also, the effect of thermal radiation on the convective flows is important in the content of space technology and processes involving high temperature. The knowledge of radiation heat transfer in the system can perhaps lead to a desired product with a sought characteristic. However, in all the stretching sheet problems (both hydrodynamic and hydromagnetic) mentioned earlier, radiation effect has not been considered. Extensive literature that deals with flows in the presence of radiation effects is now available (see e.g, England and Emery [19], Gorla and Pop [20], Raptis [21], and Abd El-Aziz [22-24]). All the above-mentioned investigations were limited to a continuous surface moving with a constant, linear, or nonlinear velocity. However very little attention is given to the flow over an exponentially stretching sheet. Magyari and Keller [25] analyzed the steady free convection flow and heat transfer from an exponentially stretching vertical surface. Elbashbeshy [26] examined the flow and heat transfer characteristics by considering exponentially stretching continuous surface. Viscoelastic boundary layer flow over an exponential stretching continuous sheet has been examined by $[27,28]$. Recently, Partha et al. [29] studied the effect of viscous dissipation on the mixed convection heat transfer from an exponentially stretching surface. Very recently, Abd El-Aziz [30] examined the problem of viscous dissipation effect on mixed convection flow of a micropolar fluid over an exponentially stretching sheet. When the conducting fluid is an ionized gas, and the strength of the applied magnetic field is large, the conductivity normal to the magnetic field is reduced due to the free spiraling of electrons and ions about the magnetic lines of force before suffering collisions and a current is induced in a direction normal to both electric and magnetic fields. This phenomenon is called Hall effect. In all of the previous investigations, the Hall term was ignored in applying Ohm's law as it has no marked effect 
for small and moderate values of the magnetic field. When the medium is rarefied or if a strong magnetic field is present, the conductivity of the fluid is anisotropic and the effect of Hall current cannot be neglected. The study of MHD viscous flows with Hall current has important applications in problems of power generators and Hall accelerators as well as flight magnetohydrodynamics. The current trend for the application of magnetohydrodynamics is toward a strong magnetic field (so that the influence of electromagnetic force is noticeable) and towards a low density of the gas (such as in space flight and in nuclear fusion research) [31]. Under these conditions the Hall current becomes important. With the above understanding, Pop and Watanabe [32] studied the problem of free convection flow of an electrically conducting viscous fluid without neglecting the Hall effect. Abo-Eldahab and Abd El-Aziz [33] analyzed the problem of MHD free convection flow of an electrically conducting and heat generating/absorbing fluid past a semi-infinite vertical plate taking into consideration the effects of Hall and ion-slip currents. Megahed et al. [34] investigated the effects of Hall currents on a steady free convection flow and mass transfer past a semi-infinite plate past a viscous incompressible electrically conducting fluid using similarity analysis. Abo-Eldahab and Abd El-Aziz [35] studied the effect of Hall current and Ohmic heating on mixed convection boundary layer flow of a micropolar fluid from a rotating cone. AboEldahab and Abd El-Aziz [36] presented an analysis for the effects of viscous dissipation and Joule heating on the flow of an electrically conducting and viscous incompressible fluid past a semi-infinite plate in the presence of a strong transverse magnetic field and heat generation/absorption. Saha et al. [37] analyzed the effect of Hall current on the steady, laminar, natural convection boundary layer flow of MHD viscous and incompressible fluid from a semi-infinite heated permeable vertical flat plate. However, relatively little work has been done on the effect of Hall current on the boundary layer flow of an electrically conducting viscous fluid past a stretching surface. The effect of Hall current on a steady, laminar, hydromagnetic boundary layer flow of an electrically conducting and heat generating/absorbing fluid along a stretching sheet is considered by Abo-Eldahab et al. [38] and Salem and Abd El-Aziz [39]. Recently, Abd El Aziz [40] studied the unsteady flow and heat transfer over a stretching surface with Hall effect. To the best of our knowledge, no analytical or numerical results have been reported for the effects of Hall current on the convective heat transfer past an exponentially stretching sheet in the presence of thermal radiation. Recently, Sajid and Hayat [41] gave analytical solution for the problem of radiation effect on heat transfer of a viscous fluid over an exponentially stretching sheet using the homotopy analysis method (HAM) (Liao [42, 43]). This method is based on a fundamental concept in topology, that is, homotopy [44] which is widely used in numerical techniques (Chan and Keller [45], Grigolyuk and Shalashilin [46]). In this paper, HAM is employed to find an analytical solution of the problem of Hall effect on MHD mixed convection flow past a vertical stretching surface in the presence of radiation.

\section{Analysis}

Consider the steady mixed convection boundary layer flow past a heated semi-infinite vertical wall stretching with velocity $U_{w}$ and a given temperature distribution $T_{w}$ moving through a quiescent viscous, incompressible, and electrically conducting fluid with constant temperature $T_{\infty}$. The positive $x$ coordinate is measured along the stretching sheet in the direction of motion and the positive $y$ coordinate is measured normal to the sheet in the outward direction toward the fluid. The leading edge of the stretching sheet is taken as coincident with $z$-axis (see Figure 1). The fluid is considered to be a gray, absorbing-emitting 


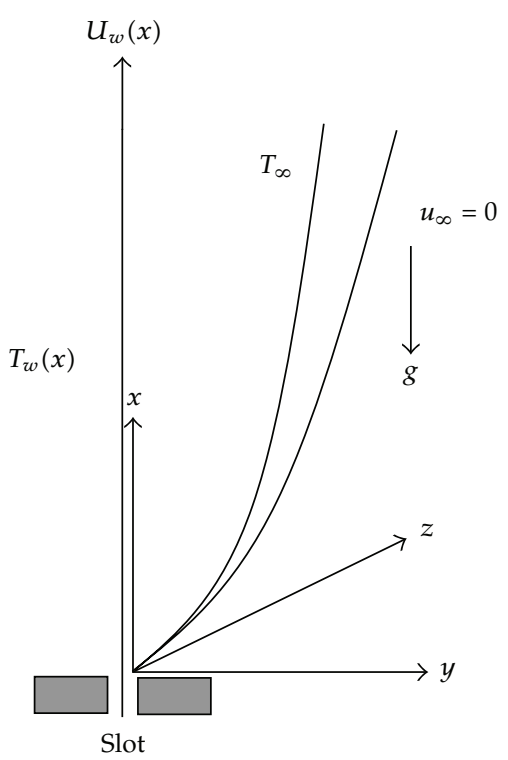

Figure 1: Schematic representation of the physical model and coordinates system.

radiation but nonscattering medium and the Rosseland approximation is used to describe the radiative heat flux in the energy equations. Also, the flow is subjected to a strong transverse magnetic field $B_{0}$ with a constant intensity along the positive $y$-direction. The magnetic Reynolds is assumed to be small enough $\left(\operatorname{Re}_{m} \ll 1\right)$ so that the induced magnetic field can be neglected. This assumption is justified since the magnetic Reynolds number is generally very small for weakly ionized gases [47]. In general, for an electrically conducting fluid, Hall current affects the flow in the presence of a strong magnetic field. The effect of Hall current gives rise to a force in the $z$-direction, which induces a crossflow in that direction and hence the flow becomes three-dimensional. To simplify the problem, we assume that there is no variation of flow quantities in $z$-direction. This assumption is considered to be valid if the surface is of infinite extent in the $z$-direction. If the Hall term is retained in generalized Ohm's law, then the following expression holds [48]:

$$
\mathbf{J}+\frac{\omega_{e} \tau_{e}}{B_{0}}(\mathbf{J} \times \mathbf{B})=\sigma\left(\mathbf{E}+\mathbf{V} \times \mathbf{B}+\frac{1}{e n_{e}} \nabla p_{e}\right)
$$

where $\mathbf{J}=\left(J_{x}, J_{y}, J_{z}\right)$ is the current density vector, $\mathbf{V}$ is the velocity vector, $\mathbf{B}=\left(0, B_{0}, 0\right)$ is the magnetic induction vector, $\omega_{e}$ is the cyclotron frequency of electrons, $\tau_{e}$ is the electron collision time, and $\sigma$ is the electrical conductivity. The ion-slip and thermoelectric effects are not included in (2.1). Further it is assumed that $\omega_{e} \tau_{e} \sim O(1)$ and $\omega_{i} \tau_{i}<1$ where $\omega_{i}$ and $\tau_{i}$ are cyclotron frequency and collision time for ions, respectively. In addition, we consider the case of a short circuit problem in which the applied electric field $\mathbf{E}=0$ and for partially ionized gas, the electron pressure gradient may be neglected. Assuming the plate to be electrically nonconducting, the generalized Ohm's law under the above conditions 
gives $J_{y}=0$ everywhere in the flow. Hence under these assumptions, equating the $x$ and $z$ components in (2.1) and solving for the current density components $J_{x}$ and $J_{z}$, we have

$$
\begin{aligned}
& J_{x}=\frac{\sigma B_{0}}{\left(1+m^{2}\right)}(m u-w), \\
& J_{z}=\frac{\sigma B_{0}}{\left(1+m^{2}\right)}(u+m w) .
\end{aligned}
$$

Here $u, v$, and $w$ are the $x-, y$-, and $z$-components of the velocity vector $\mathbf{V}$ and $m\left(=\omega_{e} t_{e}\right)$ is the Hall parameter.

Finally, we assume the fluid is isotropic, homogeneous, and has the scalar constant viscosity and electric conductivity. Under the above assumptions and invoking the Boussinesq approximation, the boundary layer equations governing the flow and heat transfer of a viscous and incompressible fluid can be written as

$$
\begin{gathered}
\frac{\partial u}{\partial x}+\frac{\partial v}{\partial y}=0 \\
u \frac{\partial u}{\partial x}+v \frac{\partial u}{\partial y}=v \frac{\partial^{2} u}{\partial y^{2}}+g \beta\left(T-T_{\infty}\right)-\frac{\sigma B_{0}^{2}}{\rho\left(1+m^{2}\right)}(u+m w), \\
u \frac{\partial w}{\partial x}+v \frac{\partial w}{\partial y}=v \frac{\partial^{2} w}{\partial y^{2}}+\frac{\sigma B_{0}^{2}}{\rho\left(1+m^{2}\right)}(m u-w), \\
u \frac{\partial T}{\partial x}+v \frac{\partial T}{\partial y}=\alpha \frac{\partial^{2} T}{\partial y^{2}}-\frac{1}{\rho c_{p}} \frac{\partial q_{r}}{\partial y}
\end{gathered}
$$

where $T$ is the fluid temperature, $v(=\mu / \rho)$ is the kinematic coefficient of viscosity with $\mu$ being the fluid viscosity and $\rho$ is the fluid density, $\alpha\left(=k / \rho c_{p}\right)$ is the thermal diffusivity with $k$ being the fluid thermal conductivity and $c_{p}$ is the heat capacity at constant pressure, and $q_{r}$ is the radiative heat flux.

The radiative heat flux $q_{r}$ under Rosseland approximation [41] has the form

$$
q_{r}=-\frac{4 \sigma}{3 k_{1}} \frac{\partial T^{4}}{\partial y}
$$

where $\sigma$ is the Stefan-Boltzmann constant and $k_{1}$ is the mean absorption coefficient.

We assume that the temperature difference within the flow is sufficiently small such that $T^{4}$ may be expressed as a linear function of temperature. This is accomplished by expanding $T^{4}$ in a Taylor series about $T_{\infty}$ and neglecting higher-order terms, thus

$$
T^{4} \cong 4 T_{\infty}^{3} T-3 T_{\infty}^{4}
$$


In view of (2.7) and (2.8), (2.6) reduces to

$$
u \frac{\partial T}{\partial x}+v \frac{\partial T}{\partial y}=\alpha \frac{\partial^{2} T}{\partial y^{2}}+\frac{16 \sigma T_{\infty}^{3}}{3 k_{1} \rho c_{p}} \frac{\partial^{2} T}{\partial y^{2}}
$$

The associated boundary conditions are

$$
\begin{aligned}
& u=U_{w}(x), \quad v=0, \quad w=0, \quad T=T_{w}(x), \quad \text { at } y=0, \\
& u \longrightarrow 0, \quad w \longrightarrow 0, \quad T \longrightarrow T_{\infty} \quad \text { at } y \longrightarrow \infty .
\end{aligned}
$$

The stretching surface is assumed to have an exponential velocity distribution of the form [30]:

$$
U_{w}(x)=U_{0} \exp \left(\frac{x}{L}\right)
$$

Here $U_{0}$ is a constant and $L$ is the reference length. The exponential velocity (2.11) is valid only when $x \ll L$ which occurs very near to the slot [28].

Also the surface temperature $T_{w}(x)$ of the stretching sheet is assumed to be in the form:

$$
T_{w}(x)=T_{\infty}+T_{0} \exp \left(\frac{a x}{2 L}\right),
$$

where $a$ and $T_{0}$ are the parameters of temperature distribution on the stretching surface and $T_{\infty}$ is the ambient temperature. The special case $a=0$ corresponds to the well-known but important particular case of the isothermal plate.

Introduce the dimensionless variables $\eta, f, h$, and $\theta$ as follows:

$$
\begin{aligned}
\eta & =y \sqrt{\frac{U_{0}}{2 v L}} \exp \left(\frac{x}{2 L}\right), \\
\psi(x, y) & =\sqrt{2 v L U_{0}} \exp \left(\frac{x}{2 L}\right) f(\eta), \\
w & =U_{0} \exp \left(\frac{x}{L}\right) h(\eta), \\
T & =T_{\infty}+\left(T_{w}-T_{\infty}\right) \theta(\eta) .
\end{aligned}
$$

In (2.14) the stream function $\psi(x, y)$ is defined by $u=\partial \psi / \partial y$ and $v=-\partial \psi / \partial x$, such that the continuity equation (2.3) is satisfied automatically and $\left(T_{w}-T_{\infty}\right)$ is given by (2.12). 
In terms of these new variables, the velocity components can be expressed as

$$
u=U_{0} \exp \left(\frac{x}{L}\right) f^{\prime}(\eta), \quad v=-\sqrt{\frac{\nu U_{0}}{2 L}} \exp \left(\frac{x}{2 L}\right)\left(f+\eta f^{\prime}\right),
$$

where prime denotes ordinary differentiation with respect to $\eta$. Now substituting (2.7)-(2.10) in $(2.4),(2.5)$, and (2.9) we obtain the following locally similar ordinary differential equations:

$$
\begin{gathered}
f^{\prime \prime \prime}+f f^{\prime \prime}-2 f^{\prime 2}+2 \xi \theta-\frac{2 M}{1+m^{2}}\left(f^{\prime}+m h\right)=0, \\
h^{\prime \prime}+f h^{\prime}-2 f^{\prime} h+\frac{2 M}{1+m^{2}}\left(m f^{\prime}-h\right)=0 \\
\left(1+\frac{4}{3 R}\right) \theta^{\prime \prime}+\operatorname{Pr}\left(f \theta^{\prime}-a f^{\prime} \theta\right)=0 .
\end{gathered}
$$

The boundary conditions (2.10) then turn into

$$
\begin{aligned}
& f^{\prime}(0)=1, \quad f(0)=0, \quad h(0)=0, \quad \theta(0)=1, \\
& f^{\prime}(\infty) \longrightarrow 0, \quad h(\infty) \longrightarrow 0, \quad \theta(\infty) \longrightarrow 0,
\end{aligned}
$$

where $\xi=G r_{x} / \operatorname{Re}_{x}^{2}$ is the mixed convection or buoyancy parameter with $G r_{x}=g \beta\left(T_{w}-\right.$ $\left.T_{\infty}\right) L^{3} / v^{2}$ being the local Grashof number and $\operatorname{Re}_{x}=U_{w} L / v$ is the local Reynolds number, $M=\sigma B_{0}^{2} L / \rho U_{w}$ is the local magnetic field parameter, $R=k k_{1} / 4 \sigma T_{\infty}^{3}$ is the radiation parameter, and $\operatorname{Pr}=v / \alpha$ is the Prandtl number.

We notice that when $\xi=0,(2.18),(2.19)$, and (2.20) are uncoupled and a purely forced convection situation results. In this case, the flow field is not affected by the thermal field. Also, in the absence of Hall $(m=0)$, buoyancy $(\xi=0)$, and radiation $(R \rightarrow \infty)$ effects, we notice that (2.18)-(2.20) reduce to those of Magyari and Keller [25].

For practical applications, the major physical quantities of interest are the local skinfriction coefficient in the $x$-direction

$$
C_{f x}=\frac{2 \tau_{w x}}{\rho U_{w}^{2}}=\frac{2 \mu(\partial u / \partial y)_{y=0}}{\rho U_{w}^{2}}=\sqrt{2} \operatorname{Re}_{x}^{-1 / 2} f^{\prime \prime}(0),
$$

local skin-friction coefficient in the $z$-direction,

$$
C_{f z}=\frac{2 \tau_{w z}}{\rho U_{w}^{2}}=\frac{2 \mu(\partial w / \partial y)_{y=0}}{\rho U_{w}^{2}}=\sqrt{2} \operatorname{Re}_{x}^{-1 / 2} h^{\prime}(0),
$$

and the local Nusselt number,

$$
N u_{x}=\frac{L}{\left(T_{w}-T_{\infty}\right)}\left(-\frac{\partial T}{\partial y}\right)_{y=0}=-\frac{1}{\sqrt{2}} \operatorname{Re}_{x}^{1 / 2} \theta^{\prime}(0)
$$




\section{HAM Solutions}

In order to solve the governing nonlinear system (2.18)-(2.20) subject to the boundary conditions (2.21) we employ the homotopy analysis method [43]. According to the boundary conditions (2.21), it is reasonable to assume that $f(\eta), h(\eta)$, and $\theta(\eta)$ can be expressed by the following set of base functions:

$$
\left\{\eta^{k} e^{-n \eta} \mid k \geqslant 0, n \geqslant 0\right\}
$$

such that

$$
\begin{aligned}
& f(\eta)=\sum_{m=0}^{\infty} \sum_{n=0}^{\infty} \sum_{k=0}^{\infty} a_{m, n}^{k} \eta^{k} e^{-n \eta}, \\
& h(\eta)=\sum_{m=0}^{\infty} \sum_{n=0}^{\infty} \sum_{k=0}^{\infty} b_{m, n}^{k} \eta^{k} e^{-n \eta}, \\
& \theta(\eta)=\sum_{m=0}^{\infty} \sum_{n=0}^{\infty} \sum_{k=0}^{\infty} d_{m, n}^{k} \eta^{k} e^{-n \eta}
\end{aligned}
$$

where $a_{m, n}^{k}, b_{m, n}^{k}$ and $d_{m, n}^{k}$ are constant coefficients. The rule of solution expression provides us with a starting point. It is under the rule of solution expression that initial approximations, auxiliary linear operators, and the auxiliary functions are determined. So, according to the rule of solution expression, we choose the initial guess and auxiliary linear operator [49-51] in the following forms:

$$
\begin{gathered}
f_{0}(\eta)=1-e^{-\eta}, \\
h_{0}(\eta)=\eta e^{-\eta}, \\
\theta_{0}(\eta)=e^{-\eta}, \\
\mathcal{L}_{f}=f^{\prime \prime \prime}+f^{\prime \prime}, \\
\mathcal{L}_{h}=h^{\prime \prime}+h^{\prime}, \\
\mathcal{L}_{\theta}=\theta^{\prime \prime}+\theta^{\prime}
\end{gathered}
$$

in which the auxiliary linear operators have the following properties:

$$
\begin{gathered}
\mathcal{L}_{f}\left[C_{1}+C_{2} \eta+C_{3} e^{-\eta}\right]=0, \\
\mathcal{L}_{h}\left[C_{4}+C_{5} e^{-\eta}\right]=0, \\
\mathcal{L}_{\theta}\left[C_{6}+C_{7} e^{-\eta}\right]=0,
\end{gathered}
$$

where $C_{i}^{\prime} s(i=1, \ldots, 7)$ are constants. Let $p \in[0,1]$ denote the embedding parameter and let $\hbar_{1}$, $\hbar_{2}$, and $\hbar_{3}$ indicate nonzero auxiliary parameters. We then construct the following equations. 


\subsection{Zeroth-Order Deformation Equations}

Consider

$$
\begin{gathered}
(1-p) \mathcal{L}_{f}\left[f(\eta ; p)-f_{0}(\eta)\right]=p \hbar_{1} N_{f}[f(\eta ; p), h(\eta ; p), \theta(\eta ; p)] \\
(1-p) \mathcal{L}_{h}\left[h(\eta ; p)-h_{0}(\eta)\right]=p \hbar_{2} N_{h}[f(\eta ; p), h(\eta ; p)] \\
(1-p) \mathcal{L}_{\theta}\left[\theta(\eta ; p)-\theta_{0}(\eta)\right]=p \hbar_{3} N_{\theta}[f(\eta ; p), \theta(\eta ; p)] \\
f(0 ; p)=0,\left.\quad \frac{d f(\eta ; p)}{d \eta}\right|_{\eta=0}=1,\left.\quad \frac{d f(\eta ; p)}{d \eta}\right|_{\eta=\infty}=0 \\
h(0 ; p)=0, \quad h(\infty ; p)=0 \\
\theta(0 ; p)=1, \quad \theta(\infty ; p)=0
\end{gathered}
$$

where the nonlinear operators $N_{f}, N_{h}$, and $N_{\theta}$, respectively, are

$$
\begin{aligned}
N_{f}[f(\eta ; p), h(\eta ; p), \theta(\eta ; p)]= & \frac{\partial^{3} f(\eta ; p)}{\partial \eta^{3}}+f(\eta ; p) \frac{\partial^{2} f(\eta ; p)}{\partial \eta^{2}} \\
& -2\left(\frac{\partial f(\eta ; p)}{\partial \eta}\right)^{2}+2 \xi \theta(\eta ; p) \\
& -\frac{2 M}{1+m^{2}}\left(\frac{\partial f(\eta ; p)}{\partial \eta}+m h(\eta ; p)\right) \\
N_{h}[f(\eta ; p), h(\eta ; p)]= & \frac{\partial^{2} h(\eta ; p)}{\partial \eta^{2}}+f(\eta ; p) \frac{\partial h(\eta ; p)}{\partial \eta}-2 \frac{\partial f(\eta ; p)}{\partial \eta} h(\eta ; p) \\
& +\frac{2 M}{1+m^{2}}\left(m \frac{\partial f(\eta ; p)}{\partial \eta}-h(\eta ; p)\right) \\
N_{\theta}[f(\eta ; p), \theta(\eta ; p)]= & (3 R+4) \frac{\partial^{2} \theta(\eta ; p)}{\partial \eta^{2}} \\
& -3 \operatorname{Pr} R\left(a \frac{\partial f(\eta ; p)}{\partial \eta} \theta(\eta ; p)-f(\eta ; p) \frac{\partial \theta(\eta ; p)}{\partial \eta}\right) .
\end{aligned}
$$


Obviously when $p=0$ and $p=1$, the above zeroth-order deformation equations (3.5) have the solutions:

$$
\begin{array}{ll}
f(\eta ; 0)=f_{0}(\eta), & f(\eta ; 1)=f(\eta), \\
h(\eta ; 0)=h_{0}(\eta), & h(\eta ; 1)=h(\eta) \\
\theta(\eta ; 0)=\theta_{0}(\eta), & \theta(\eta ; 1)=\theta(\eta) .
\end{array}
$$

Expanding $f(\eta ; p), h(\eta ; p)$, and $\theta(\eta ; p)$ in Taylor's series with respect to $p$, we have

$$
\begin{aligned}
& f(\eta ; p)=f_{0}(\eta)+\sum_{m=1}^{\infty} f_{m}(\eta) p^{m} \\
& h(\eta ; p)=h_{0}(\eta)+\sum_{m=1}^{\infty} h_{m}(\eta) p^{m} \\
& \theta(\eta ; p)=\theta_{0}(\eta)+\sum_{m=1}^{\infty} \theta_{m}(\eta) p^{m}
\end{aligned}
$$

where

$$
\begin{aligned}
& f_{m}(\eta)=\left.\frac{1}{m !} \frac{\partial^{m} f(\eta ; p)}{\partial p^{m}}\right|_{p=0}, \\
& h_{m}(\eta)=\left.\frac{1}{m !} \frac{\partial^{m} h(\eta ; p)}{\partial p^{m}}\right|_{p=0}, \\
& \theta_{m}(\eta)=\left.\frac{1}{m !} \frac{\partial^{m} \theta(\eta ; p)}{\partial p^{m}}\right|_{p=0} .
\end{aligned}
$$

\section{2. mth-Order Deformation Equations}

Differentiating the zeroth-order deformation equations (3.5) $m$-times with respect to $p$, then setting $p=0$, and finally dividing them by $m$ !, we obtain the $m$ th-order deformation equations:

$$
\begin{aligned}
& \mathcal{L}_{f}\left[f_{m}(\eta)-x_{m} f_{m-1}(\eta)\right]=\hbar_{1} R_{m}^{f}(\eta), \\
& \mathcal{L}_{h}\left[h_{m}(\eta)-x_{m} h_{m-1}(\eta)\right]=\hbar_{2} R_{m}^{h}(\eta), \\
& \left\llcorner_{\theta}\left[\theta_{m}(\eta)-x_{m} \theta_{m-1}(\eta)\right]=\hbar_{3} R_{m}^{\theta}(\eta)\right.
\end{aligned}
$$


subject to the boundary conditions:

$$
\begin{gathered}
f_{m}(0)=\left.\frac{d f_{m}(\eta ; 0)}{d \eta}\right|_{\eta=0}=\left.\frac{d f_{m}(\eta ; 0)}{d \eta}\right|_{\eta=\infty}=0, \\
h_{m}(0)=h_{m}(\infty)=0, \\
\theta_{m}(0)=\theta_{m}(\infty)=0,
\end{gathered}
$$

where

$$
\begin{gathered}
R_{m}^{f}(\eta)=f_{m-1}^{\prime \prime \prime}(\eta)+2 \xi \theta_{m-1}(\eta)-\frac{2 M}{1+m^{2}}\left(f_{m-1}^{\prime}(\eta)+m h_{m-1}(\eta)\right) \\
+\sum_{k=0}^{m-1}\left[f_{m-1-k}(\eta) f_{k}^{\prime \prime}(\eta)-2 f_{m-1-k}^{\prime}(\eta) f_{k}^{\prime}(\eta)\right] \\
R_{m}^{h}(\eta)=h_{m-1}^{\prime \prime}(\eta)+\frac{2 M}{1+m^{2}}\left(m f_{m-1}^{\prime}(\eta)-h_{m-1}(\eta)\right) \\
+\sum_{k=0}^{m-1}\left[f_{m-1-k}(\eta) h_{k}^{\prime}(\eta)-2 h_{m-1-k}(\eta) f_{k}^{\prime}(\eta)\right] \\
R_{m}^{\theta}(\eta)=(3 R+4) \theta_{m-1}^{\prime \prime}(\eta)-3 \operatorname{Pr} R \sum_{k=0}^{m-1}\left[a \theta_{m-1-k}(\eta) f_{k}^{\prime}(\eta)-\theta_{m-1-k}^{\prime}(\eta) f_{k}(\eta)\right] \\
X_{m}= \begin{cases}0, & m \leqslant 1 \\
1, & m>1 .\end{cases}
\end{gathered}
$$

If we let $f_{m}^{*}(\eta), h_{m}^{*}(\eta)$, and $\theta_{m}^{*}(\eta)$ as the special solutions of (3.11), the general solution is given by

$$
\begin{gathered}
f_{m}(\eta)=f_{m}^{*}(\eta)+C_{1}+C_{2} \eta+C_{3} e^{-\eta}, \\
h_{m}(\eta)=h_{m}^{*}(\eta)+C_{4}+C_{5} e^{-\eta}, \\
\theta_{m}(\eta)=\theta_{m}^{*}(\eta)+C_{6}+C_{7} e^{-\eta},
\end{gathered}
$$

where the integral constants $C_{i}^{\prime} s(i=1, \ldots, 7)$ are determined by the boundary conditions (3.12). In this way it is easy to solve the linear nonhomogeneous equations (3.11) by using Maple one after the other in the order $m=1,2,3, \ldots$

\subsection{Convergence of the Analytic Solution}

Liao [43] showed that whenever a solution series converges, it will be one of the solutions of considered problem. Therefore, it is important to ensure that the solutions series are convergent. The solutions series (3.9) contain the nonzero auxiliary parameters $\hbar_{1}, \hbar_{2}$, and $\hbar_{3}$, which can be chosen properly by plotting the so-called $\hbar$-curves to ensure the convergence of 


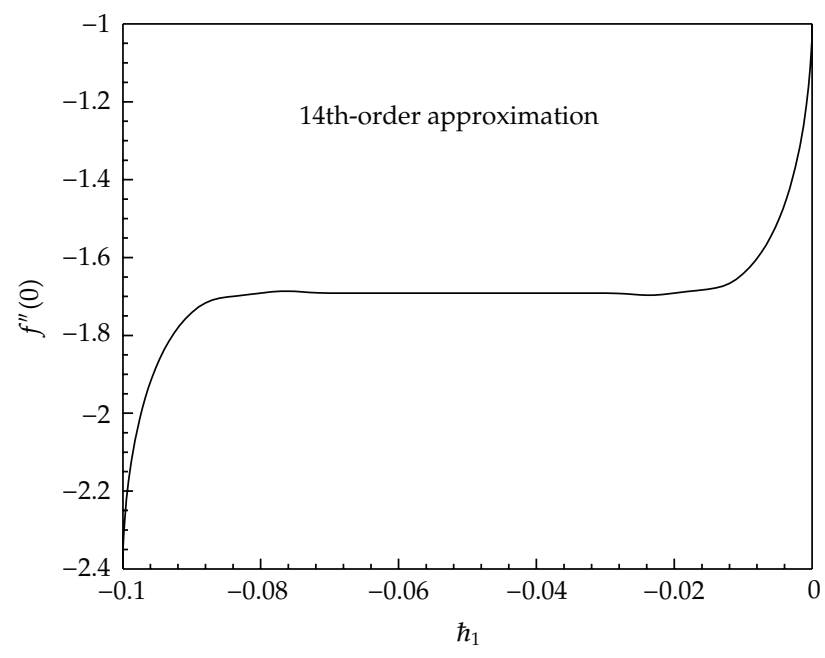

Figure 2: $\hbar_{1}$ curve for 14th-order of approximation.

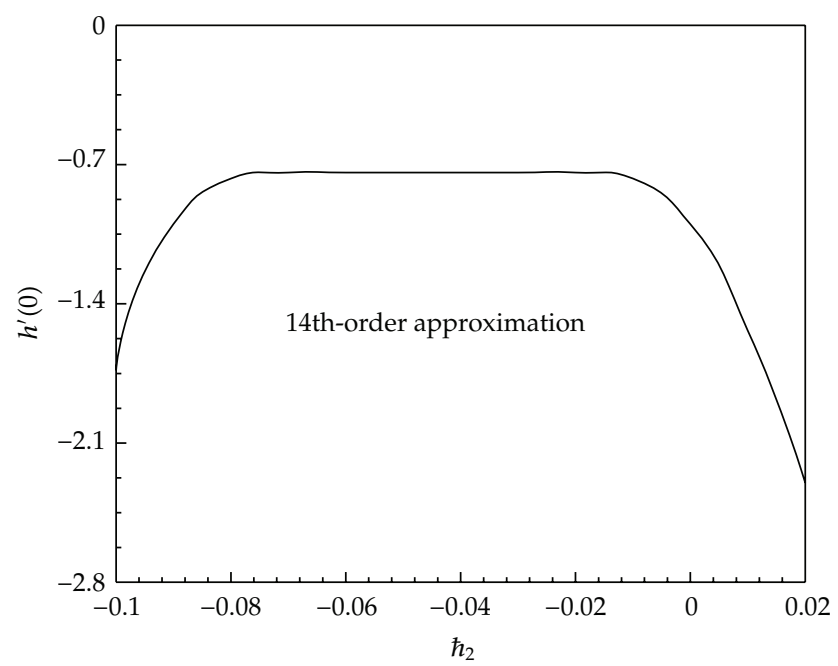

Figure 3: $\hbar_{2}$ curve for 14 th-order of approximation.

the solutions series and rate of approximation of the HAM solution. To plot the convergence curve of one of the auxiliary parameters $\hbar_{1}, \hbar_{2}$, and $\hbar_{3}$ we must choose the values of other two parameters. In the present work, the optimal HAM [52] is used to obtain the optimal values of the auxiliary parameters by means of the minimum of the residual squares of the governing equations. The interval on $\hbar$-axis for which the $\hbar$-curve becomes parallel to the $\hbar$ axis is recognized as the set of admissible values of $\hbar$ for which the solution series converges. To see the range for admissible values of $\hbar_{1}, \hbar_{2}$, and $\hbar_{3}$ for the present problem, $\hbar$-curves of $f^{\prime \prime}(0), h^{\prime}(0)$, and $\theta^{\prime}(0)$ are shown in Figures 2, 3, and 4 for 14th-order of approximation when $\operatorname{Pr}=0.72, M=n=1, m=0.2, R=5$, and $\xi=1$. According to these figures, the convergence ranges for $f^{\prime \prime}(0), h^{\prime}(0)$, and $\theta^{\prime}(0)$ are $-0.07 \leqslant \hbar_{1} \leqslant-0.01,-0.07 \leqslant$ $\hbar_{2} \leqslant-0.01$, and $-0.1 \leqslant \hbar_{3} \leqslant-0.7$. To assure the convergence of the HAM solution, the values of $\hbar_{1}, \hbar_{2}$, and $\hbar_{3}$ should be chosen from these regions. The region for the 


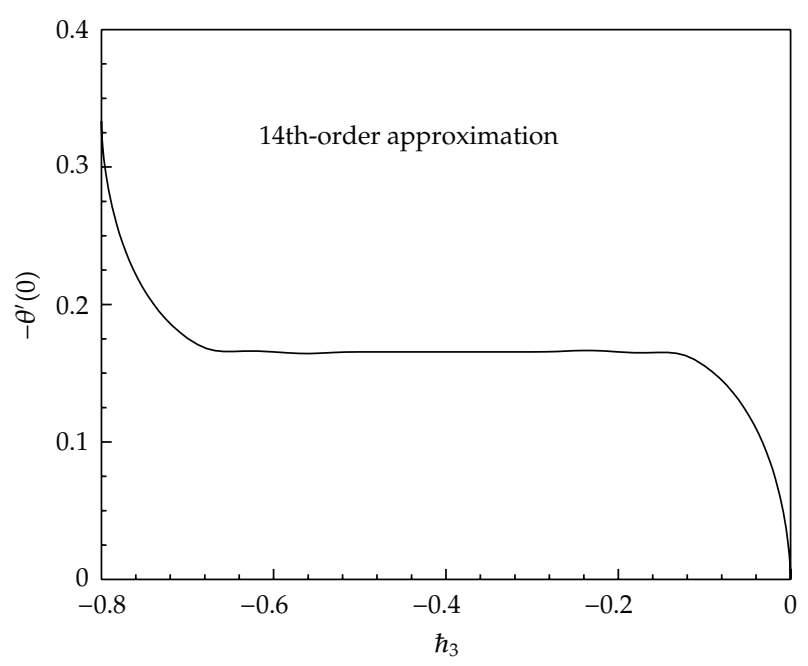

Figure 4: $\hbar_{3}$ curve for 14 th-order of approximation.

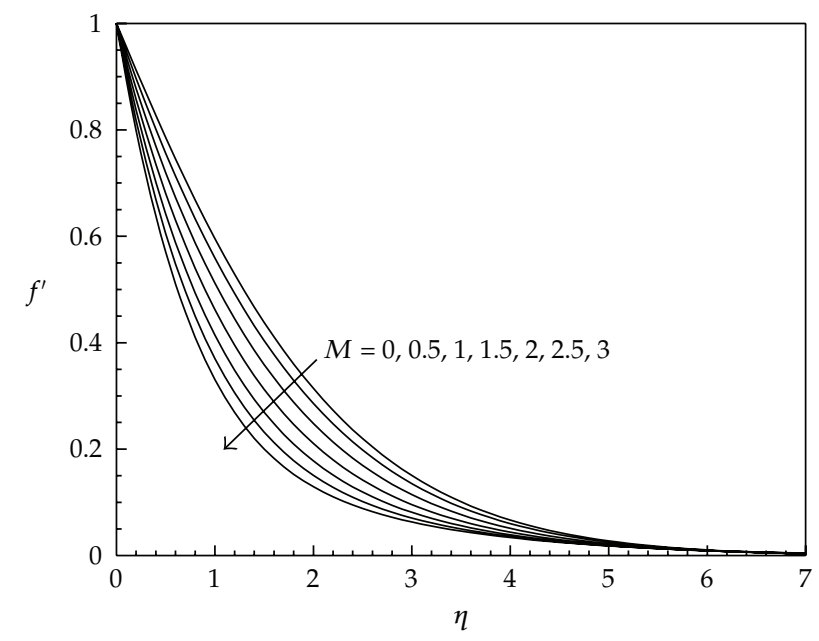

Figure 5: Tangential velocity profiles for various values of $M$ with $m=2, \xi=1, a=1, R=5$, and $\operatorname{Pr}=0.72$.

values of $\hbar_{1}, \hbar_{2}$, and $\hbar_{3}$ is strongly dependent on the values of involving parameters. Obviously our calculations show that the series (3.9) converge in the whole region of $\eta$ when $\hbar_{1}=\hbar_{2}=-0.04$ and $\hbar_{3}=-0.4$.

\section{Results and Discussion}

This section describes the graphical results of some interesting parameters for velocity and temperature profiles. Figures 5, 6, and 7 present typical tangential velocity $f^{\prime}(\eta)$, transverse velocity $h(\eta)$, and temperature $\theta(\eta)$ profiles for $m=0.2, \xi=1, a=1, R=5$, and $\operatorname{Pr}=0.72$ and various values of the magnetic parameter $M(M=0,0.5,1,1.5,2,2.5$ and 3). Figures 5 and 7 demonstrate that the velocity of the fluid diminishes, whilst the temperature distribution 


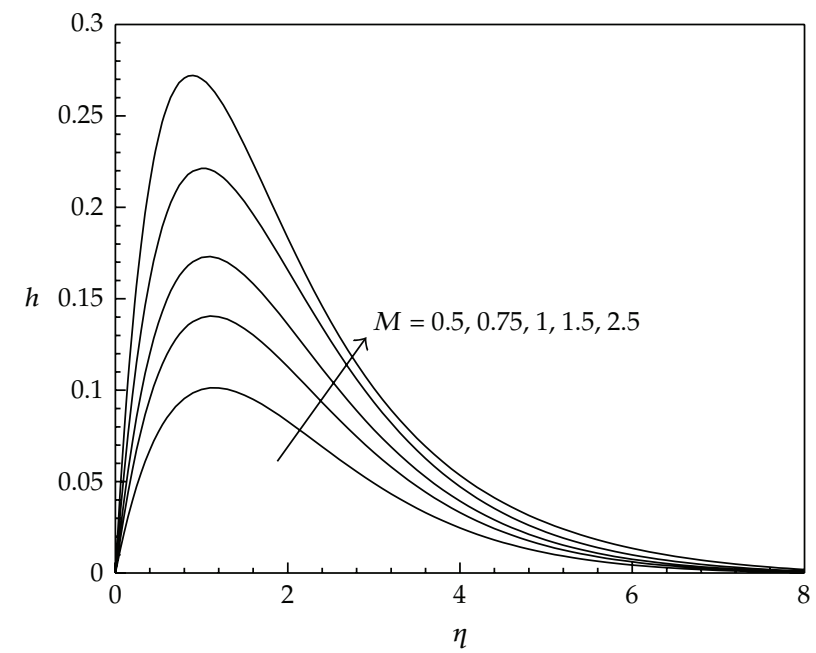

Figure 6: Transverse velocity profiles for various values of $M$ with $m=2, \xi=1, a=1, R=5$, and $\operatorname{Pr}=0.72$.

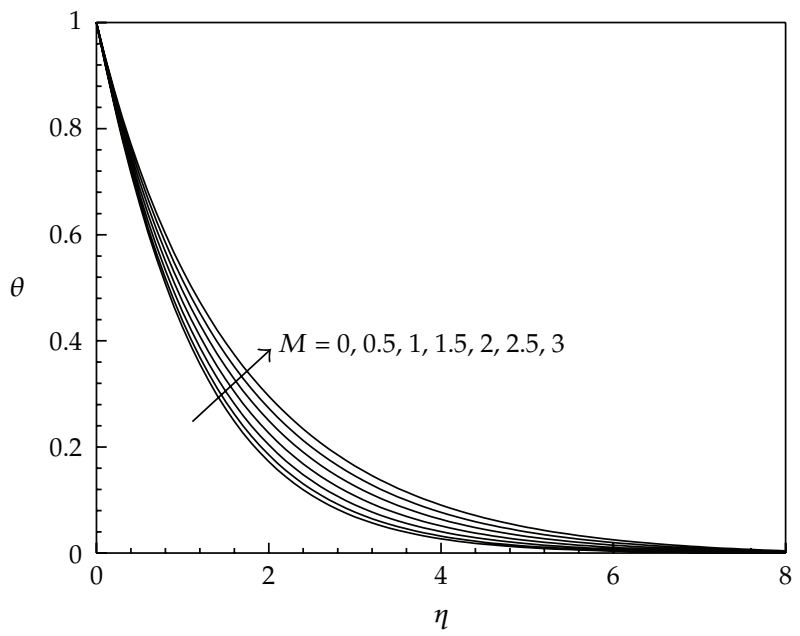

Figure 7: Temperature profiles for various values of $M$ with $m=2, \xi=1, a=1, R=5$, and $\operatorname{Pr}=0.72$.

enhances within the boundary layer the magnetic parameter $M$ rising from 0 to 3 . This is due to the fact that the application of a transverse magnetic field results in a drag-like force called the Lorentz force. This force tends to slow down the movement of the fluid along surface and increases in the temperature. On the other hand, as $M$ increases a crossflow in the transverse direction is greatly induced due to the Hall effect. Accordingly the transverse velocity $h(\eta)$ increases as $M$ increases as shown in Figure 6.

Figures 8,9 , and 10 illustrate the influence of the Hall parameter $m$ on the tangential velocity $f^{\prime}(\eta)$, transverse velocity $h(\eta)$, and temperature $\theta(\eta)$ profiles in the boundary layer. Figure 8 shows that the tangential velocity $f^{\prime}(\eta)$ increases while the temperature $\theta(\eta)$ decreases with increasing $m$. This is due to the fact that the effective conductivity $\left(\sigma /\left(1+m^{2}\right)\right)$ decreases with increasing $m$ which in turn reduces the magnetic damping force on $f^{\prime}(\eta)$. Also it is shown from these figures that the velocity and temperature profiles approach their 


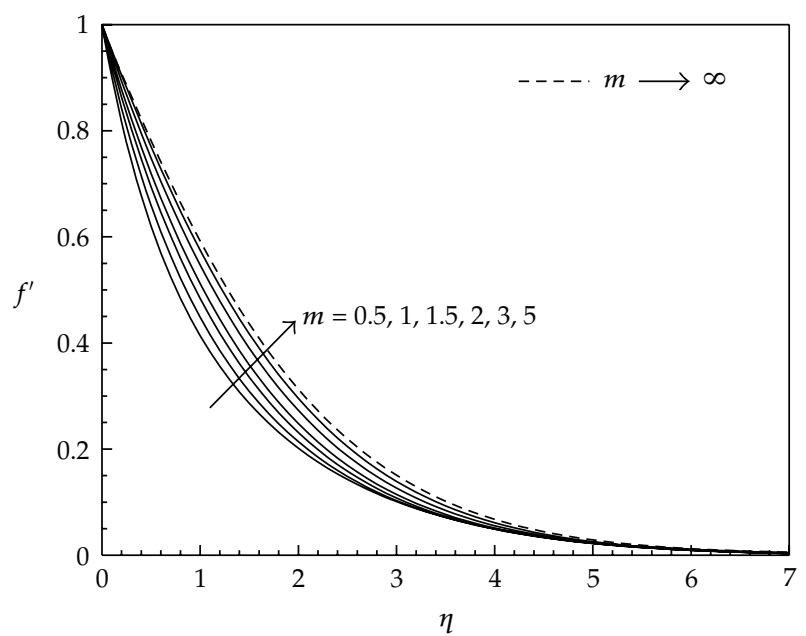

Figure 8: Tangential velocity profiles for various values of $m$ with $M=1, \xi=1, a=1, R=5$, and $\operatorname{Pr}=0.72$.

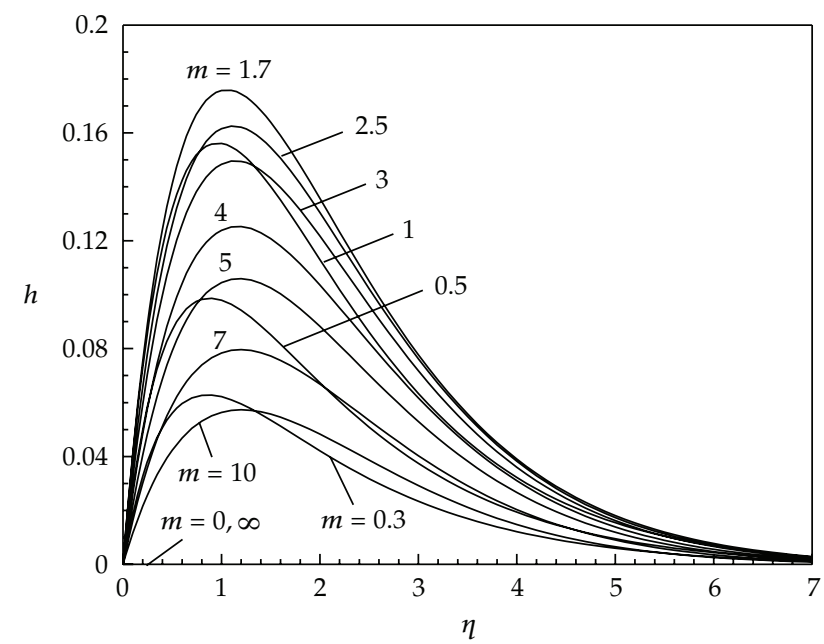

Figure 9: Transverse velocity profiles for various values of $m$ with $M=1, \xi=1, a=1, R=5$, and $\operatorname{Pr}=0.72$.

classical hydrodynamic values when the Hall parameter $m$ increases to $\infty$ since the magnetic force terms approach zero value for very large values of $m$. On the other hand Figure 9 shows that the transverse flow in the $z$-direction first increases gradually with $m$, reaching a maximum profile for $m=1.7$ and then decreases for $m>1.7$ being equal to zero when $m$ becomes very large. This is due to the fact that for large values of $m$, the term $\left(1 /\left(1+m^{2}\right)\right)$ is very small and hence the resistive effect of the magnetic field is diminished.

In Figures 11, 12, and 13, the influence of the radiation parameter $R$ on the profiles of the tangential velocity, transverse velocity, and temperature is presented, respectively, for $M=1, m=0.2, \xi=1, a=1$, and $\operatorname{Pr}=0.72$. From Figure 13 it is obvious that the temperature $\theta(\eta)$ is greatly increased as $R$ is decreased. This is due to the fact that a decrease in the values of $R\left(=k k_{1} / 4 \sigma T_{\infty}^{3}\right)$ for given $k$ and $T_{\infty}$ leads to a decrease in the Rosseland radiation absorptivity $k_{1}$. According to (2.6) and (2.7), the divergence of the radiative heat flux $\partial q_{r} / \partial y$ increases as $k_{1}$ decreases which in turn increases the rate of radiative heat transferred to 


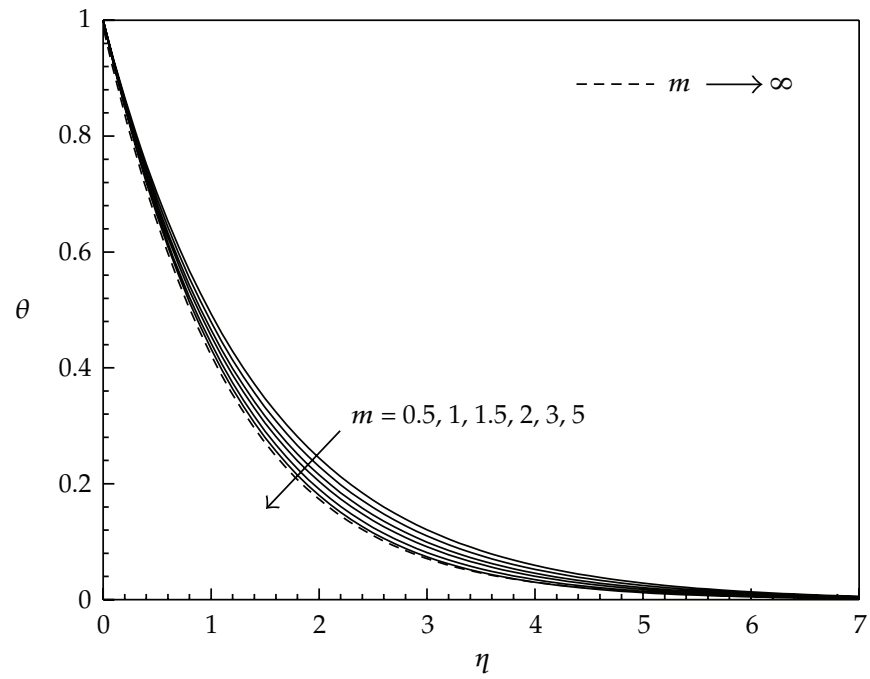

Figure 10: Temperature profiles for various values of $m$ with $M=1, \xi=1, a=1, R=5$, and $\operatorname{Pr}=0.72$.

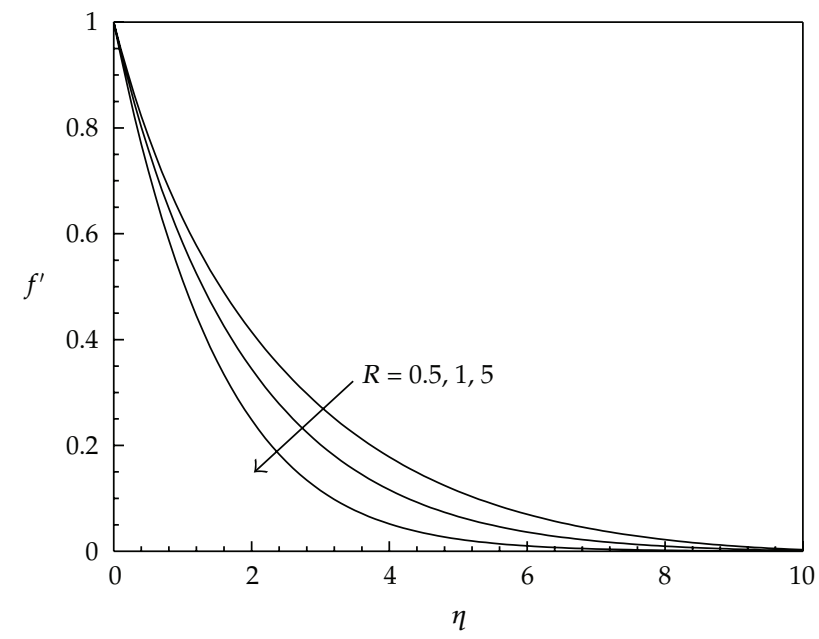

Figure 11: Tangential velocity profiles for various values of $R$ with $M=1, m=2, \xi=1, a=1$, and $\operatorname{Pr}=$ 0.72 .

the fluid and hence the fluid temperature increases. In view of this explanation, the effect of radiation becomes more pronounced as $R \rightarrow 0(R \neq 0)$ and can be ignored when $R \rightarrow \infty$. The increase in the fluid temperature has a direct effect on the buoyancy force which in turn induces more flow in the boundary layer causing the velocity (tangential and transverse) of the fluid there to increase as obvious from Figures 11 and 12. Also, it is seen from Figures 11-13 that the larger the $R$, the thinner the momentum and thermal boundary layer thickness.

The effect of the buoyancy parameter $\xi$ on the tangential velocity $f^{\prime}(\eta)$, transverse velocity $h(\eta)$, and temperature $\theta(\eta)$ profiles is displayed in Figures 14, 15, and 16, respectively, for $M=1, m=0.2, a=1, R=5$, and $\operatorname{Pr}=0.72$. From Figure 14 it is seen that the tangential velocity $f^{\prime}(\eta)$ increases with the positive values (assisting) of the buoyancy parameter $\xi$ and decreases with the negative values (opposing flow) of $\xi$ as compared to the case of pure forced convection $(\xi=0)$. This is due to the fact that a positive $\xi$ induces 


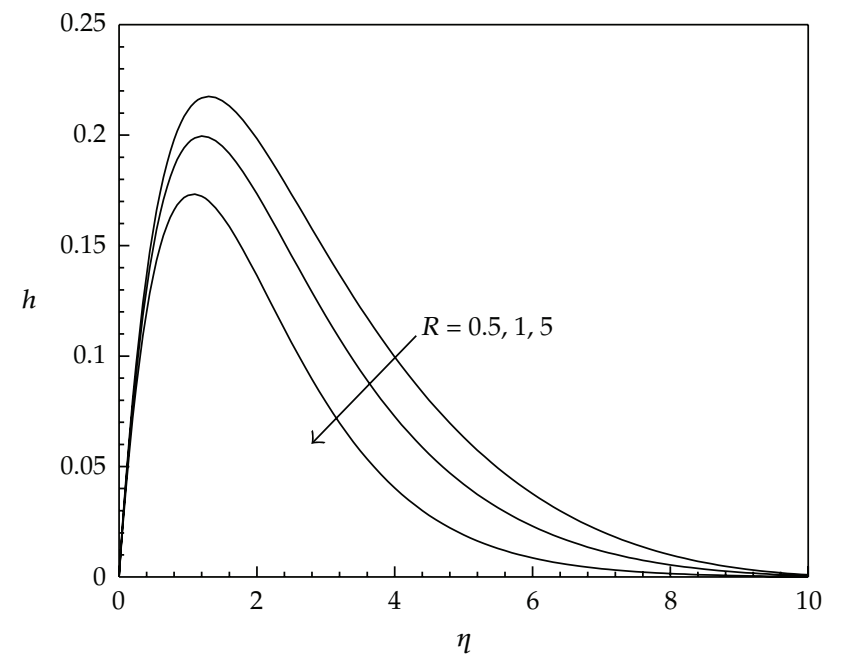

Figure 12: Transverse velocity profiles for various values of $R$ with $M=1, m=2, \xi=1, a=1$, and $\operatorname{Pr}=$ 0.72 .

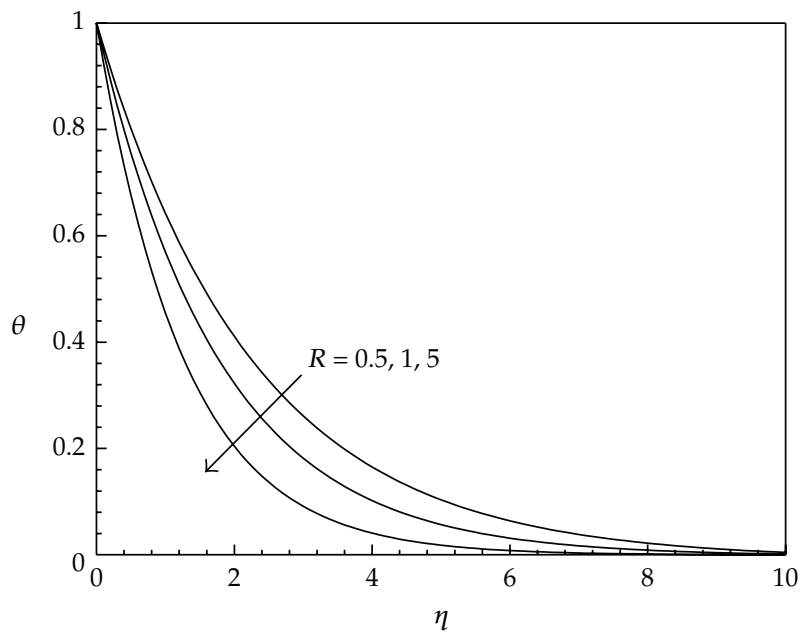

Figure 13: Temperature profiles for various values of $R$ with $M=1, m=2, \xi=1, a=1$, and $\operatorname{Pr}=0.72$.

a favorable pressure gradient that enhances the fluid flow in the boundary layer, while a negative $\xi$ produces an adverse pressure gradient that slows down the fluid motion. The effect of buoyancy parameter $\xi$ on the transverse velocity $h(\eta)$ is the same as that on the tangential velocity $f^{\prime}(\eta)$ as shown in Figure 15. Further, it is clear from Figure 16 that the effect of buoyancy parameter $\xi$ is to increase the temperature $\theta(\eta)$ in the case of opposing flow and decrease it in the case of assisting flow. The reason for this trend is that the positive (negative) buoyancy force accelerates (decelerates) the fluid in the boundary layer (as mentioned earlier) which results in thinner (thicker) thermal boundary layer.

The effect of the wall temperature distribution characterized by the parameter $a$ on the profiles of $f^{\prime}(\eta), h(\eta)$, and $\theta(\eta)$ is depicted in Figures 17,18 , and 19 for $M=1, m=0.2$, $\xi=1, R=5$, and $\operatorname{Pr}=0.72$. It is seen from Figure 19 that the temperature $\theta(\eta)$ decreases as $a$ increases. The decreased temperature has a direct effect in decreasing the thermal buoyancy 


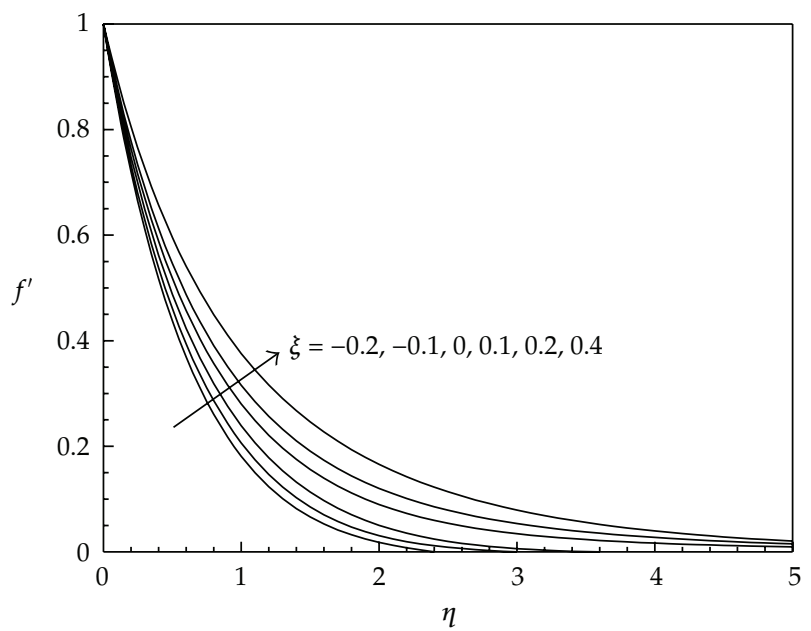

Figure 14: Tangential velocity profiles for various values of $\xi$ with $M=1, m=2, a=1, R=5$, and $\operatorname{Pr}=$ 0.72 .

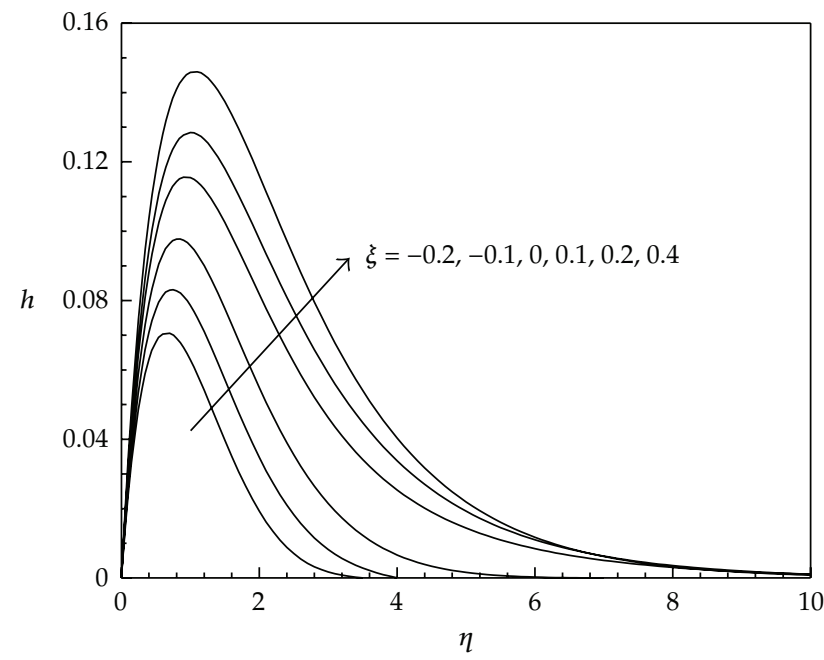

Figure 15: Transverse velocity profiles for various values of $\xi$ with $M=1, m=2, a=1, R=5$, and $\operatorname{Pr}=$ 0.72 .

forces, which in turn decrease the tangential and lateral velocities $f^{\prime}(\eta)$ and $h(\eta)$, respectively, as obvious from Figures 17 and 18. Also, Figure 19 shows a change in the direction of heat flow as a result of the peak which occurs in the temperature profile when $a=-1.5$. The presence of the peak indicates that the temperature attains its maximum value in the body of the fluid close to the surface and not at the surface and hence heat is expected to transfer to the surface from the ambient fluid.

The variations with positive values of the buoyancy parameter $\xi(0 \leqslant \xi \leqslant 2)$ of the local skin-friction coefficient in the $x$-direction in terms of $f^{\prime \prime}(0)$, the local skin-friction coefficient in the $z$-direction in terms of $h^{\prime}(0)$, and the local Nusselt number in terms of $-\theta^{\prime}(0)$ for various values of the radiation parameter $R$ and the wall temperature distribution 


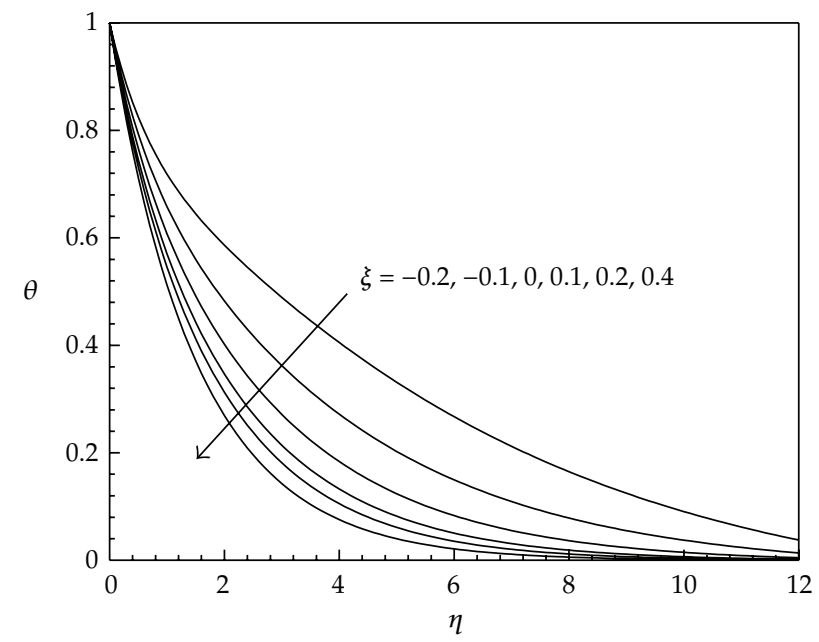

Figure 16: Temperature profiles for various values of $\xi$ with $M=1, m=2, a=1, R=5$, and $\operatorname{Pr}=0.72$.

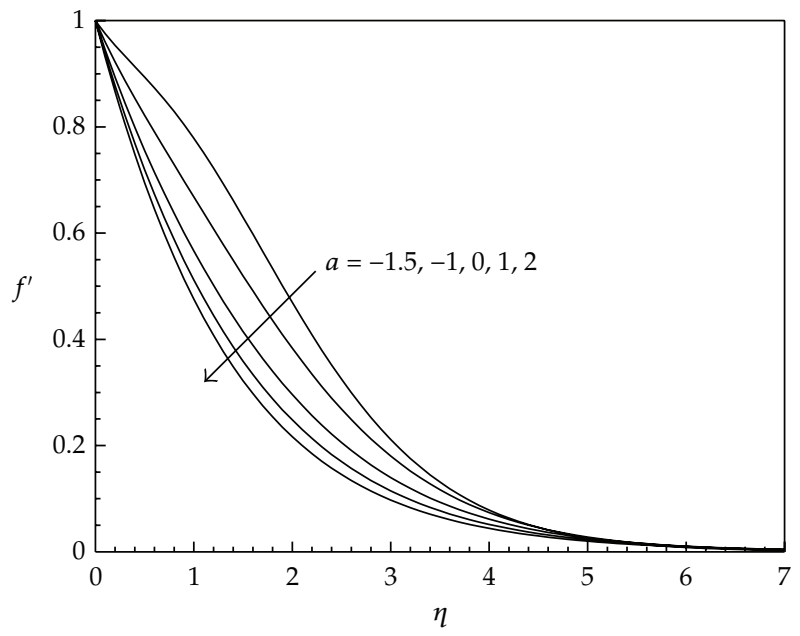

Figure 17: Tangential velocity profiles for various values of $a$ with $M=1, m=2, \xi=1, R=5$, and $\operatorname{Pr}=$ 0.72 .

characterized by the parameter $a$ are shown in Figures 20, 21, and 22, respectively. Figure 20 shows that for a fixed $\xi$ and both values of $R$, the local skin-friction coefficient $f^{\prime \prime}(0)$ increases for negative values of $a(a<0)$ and decreases for positive values of $a(a>0)$ as compared to the constant wall temperature case $(a=0)$. Also, the effect of $a$ on $f^{\prime \prime}(0)$ is more pronounced for higher values of $\xi$. In addition, for given $R$ and $a$ the local skin-friction coefficient $f^{\prime \prime}(0)$ is greatly increased as $\xi$ is increased. As mentioned earlier, the positive buoyancy force acts like a favorable pressure gradient which accelerates the fluid in the boundary layer. This results in thinner boundary layer and hence in higher velocity gradient at the surface. Therefore, the skin friction coefficient increases with $\xi$. Further, it is noted from Figure 20 that a single value of $f^{\prime \prime}(0)=-1.4737$ is obtained for all $R$ and $a$ values when $\xi=0$ (the forced convection flow). This is because (2.18) and (2.20) are uncoupled when $\xi=0$; that is, 


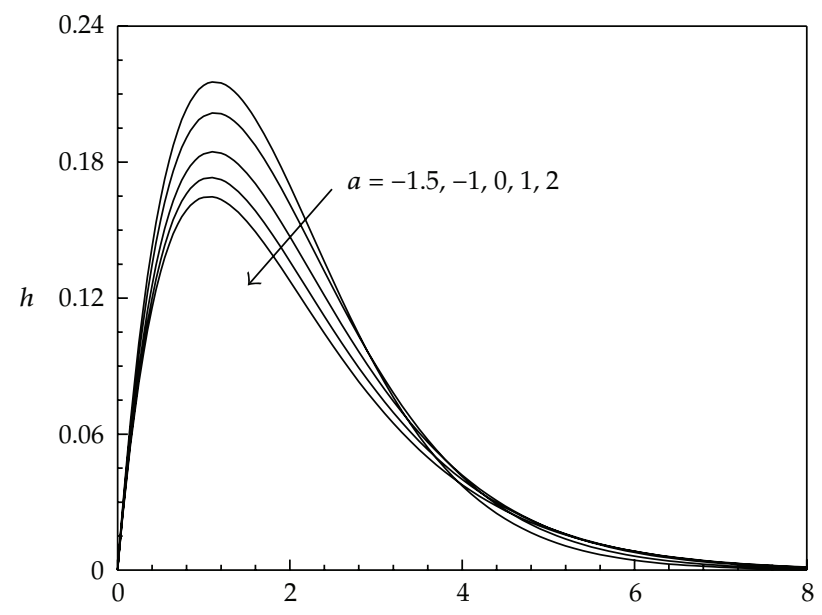

Figure 18: Transverse velocity profiles for various values of $a$ with $M=1, m=2, \xi=1, R=5$, and $\operatorname{Pr}=$ 0.72 .

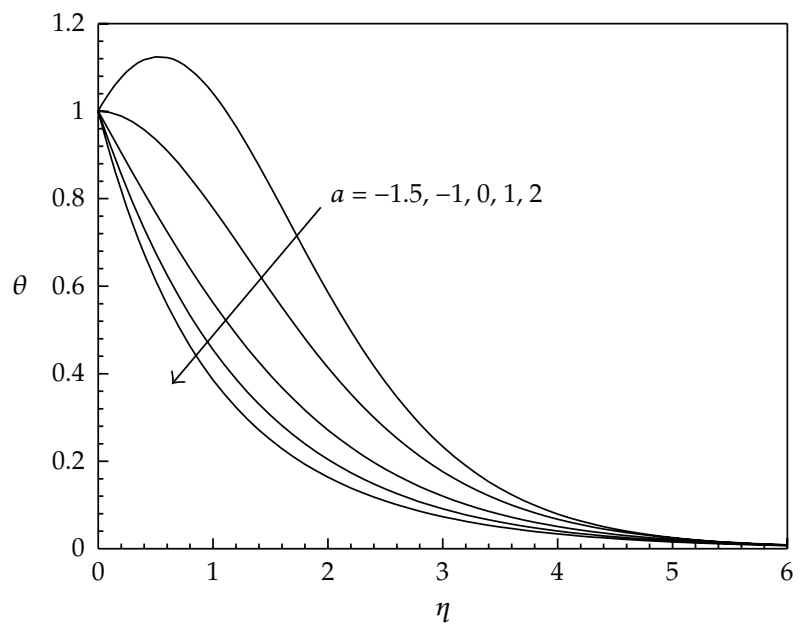

Figure 19: Temperature profiles for various values of $a$ with $M=1, m=2, \xi=1, R=5$, and $\operatorname{Pr}=0.72$.

the solutions of the flow and thermal fields are independent when buoyancy force is absent. Accordingly the parameters of the thermal field have no effect on the flow field. On the other hand, Figure 20 shows for given $\xi(\xi>0)$ that $f^{\prime \prime}(0)$ decreases with increasing $R$ for all values of $a$ but the effect of $R$ is more pronounced for positive values of $a$ and larger values of $\xi$.

The effects of $\xi, R$, and $a$ on the local skin-friction coefficient in the $z$-direction $h^{\prime}(0)$ are the same as those on $f^{\prime \prime}(0)$ as depicted in Figure 21. It is seen further that $h^{\prime}(0)$ decreases with $R$ for all $a$-values but this trend is found to be more noticeable at a positive value of $a$ and larger values of $\xi$. On the other hand, for forced convection flow $(\xi=0)$, the local skin-friction coefficient $h^{\prime}(0)$ has a unique value of 0.322377 despite the values of $R$ and $a$ for the same reason mentioned previously.

Figure 22 reveals that the local Nusselt number $-\theta^{\prime}(0)$ is increased with increasing the value of $a$ for all values of $R$ and $\xi$; in other words, heat transfer rate can be enhanced by enlarging the surface temperature variation. Also, the local Nusselt number $-\theta^{\prime}(0)$ increases 


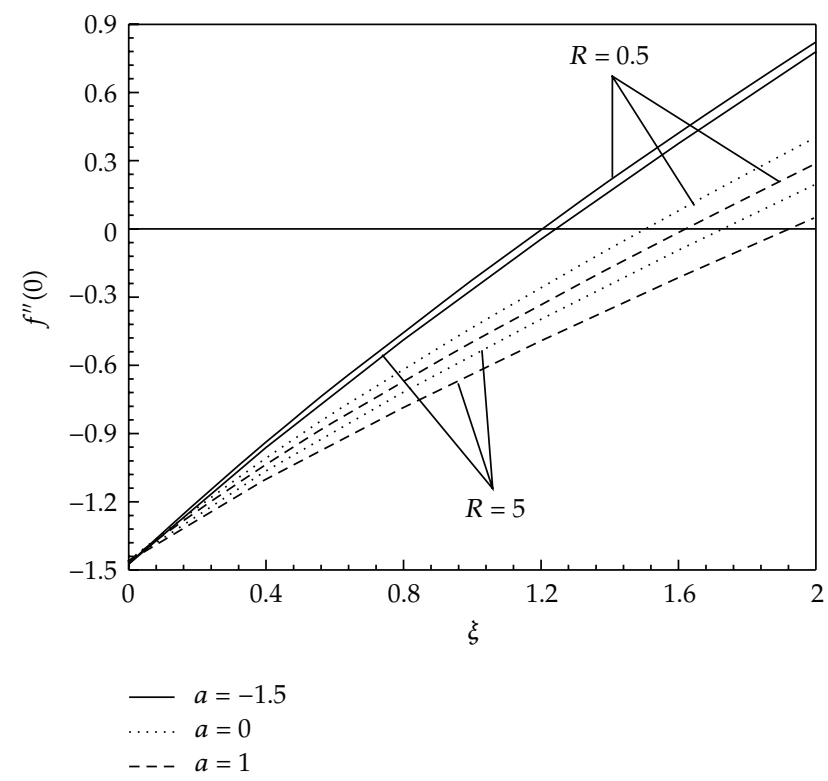

Figure 20: Local skin-friction coefficient $f^{\prime \prime}(0)$ for various values of $R$ and $a$ for $M=1, m=2, \xi=1$, and $\operatorname{Pr}=0.72$.

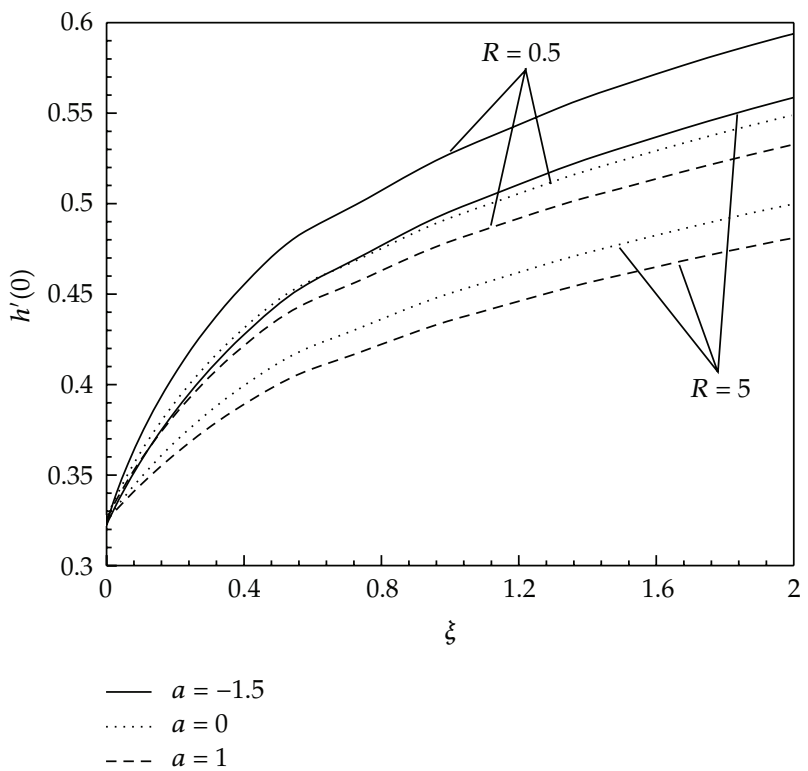

Figure 21: Local skin-friction coefficient $h^{\prime}(0)$ for various values of $R$ and $a$ for $M=1, m=2, \xi=1$, and $\operatorname{Pr}=0.72$.

with $R$ for all $\xi$ and a negative value of $a(a=-1.5)$. On the other hand, the effect of radiation parameter $R$ on $-\theta^{\prime}(0)$ for a constant wall temperature $(a=0)$ and a positive value of $a(a=1)$ is opposite to that of negative value of $a$. Namely, the heat transfer rate is reduced as $R$ is increased for a positive value of $a$ and all values of $\xi$. It is noted that negative heat transfer rates are obtained for $a=-1.5$ which indicate that heat is transferred from the fluid 


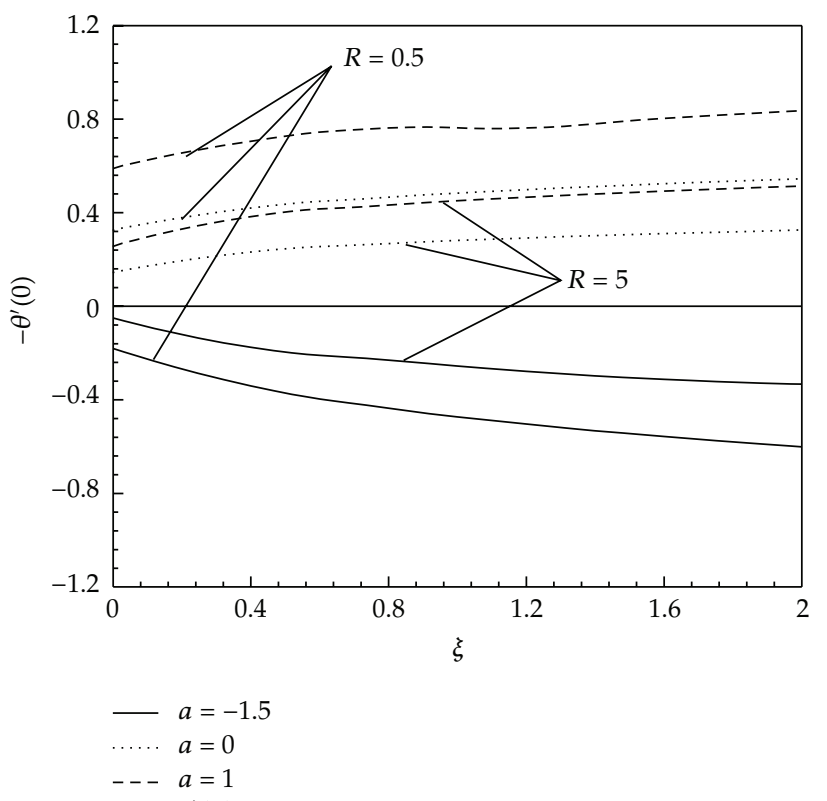

Figure 22: Local Nusselt number $-\theta^{\prime}(0)$ for various values of $R$ and $a$ for $M=1, m=2, \xi=1$, and $\operatorname{Pr}=$ 0.72 .

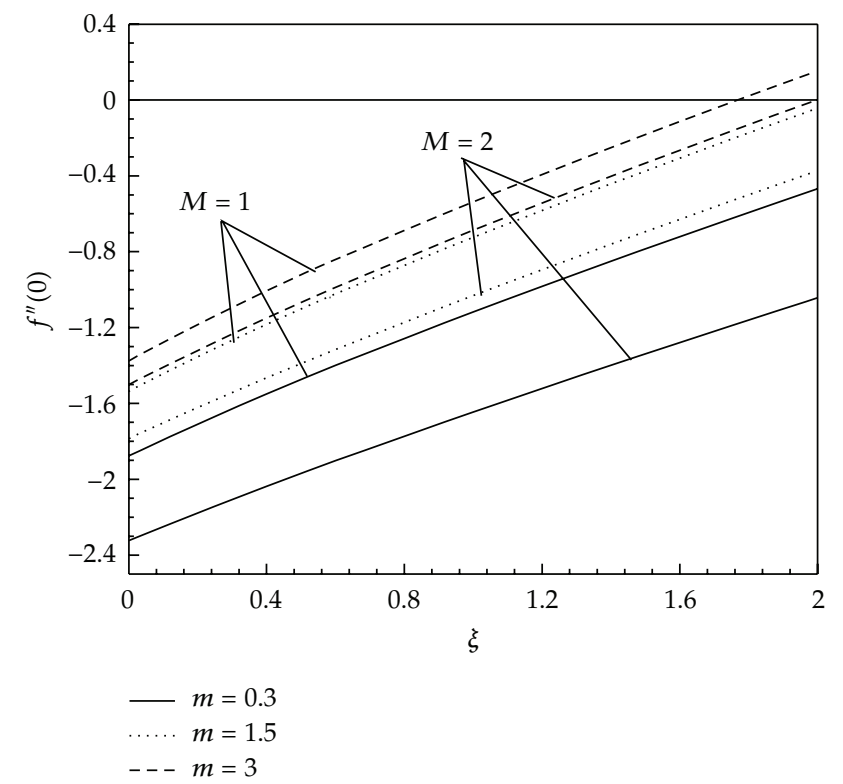

Figure 23: Local skin-friction coefficient $f^{\prime \prime}(0)$ for various values of $M$ and $m$ for $\xi=1, R=5, a=1$, and $\operatorname{Pr}=0.72$.

to the stretching surface in spite of the excess of surface temperature over that of the free stream fluid. On the other hand, $-\theta^{\prime}(0)$ increases with $\xi$ for values of $a \geqslant 0$ while the opposite trend is true for $a<0$.

The variations of the local skin-friction coefficient in the $x$-direction $f^{\prime \prime}(0)$, the local skin-friction coefficient in the $z$-direction $h^{\prime}(0)$, and the local Nusselt number $-\theta^{\prime}(0)$ as a function of the buoyancy parameter $\xi$ for two $M$ values of 1 and 2 and different values of Hall parameter $m$ are presented in Figures 23, 24, and 25. It is clear from these figures that 


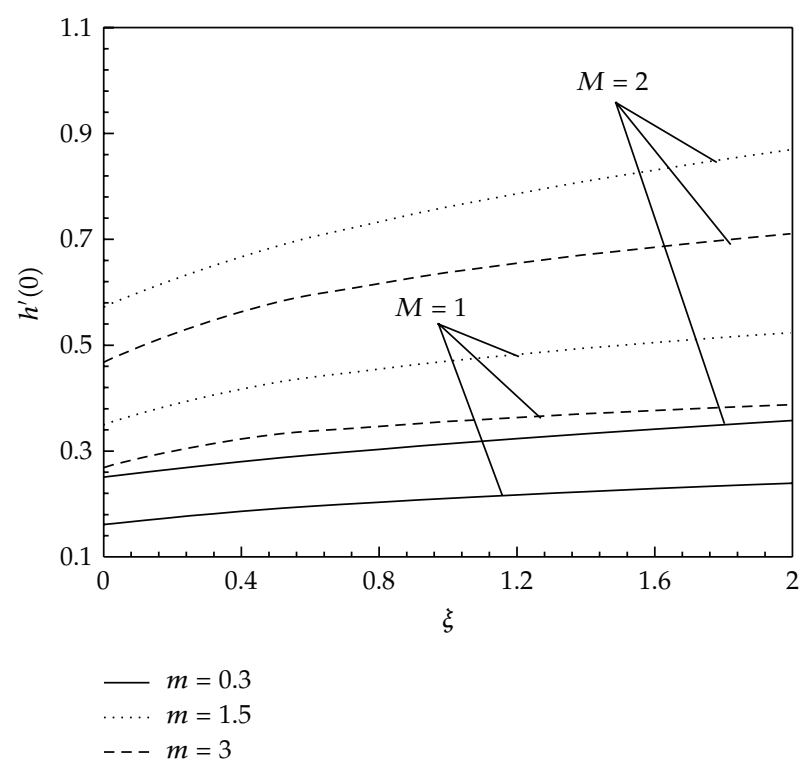

Figure 24: Local skin-friction coefficient $h^{\prime}(0)$ for various values of $M$ and $m$ for $\xi=1, R=5, a=1$ and $\operatorname{Pr}=0.72$.

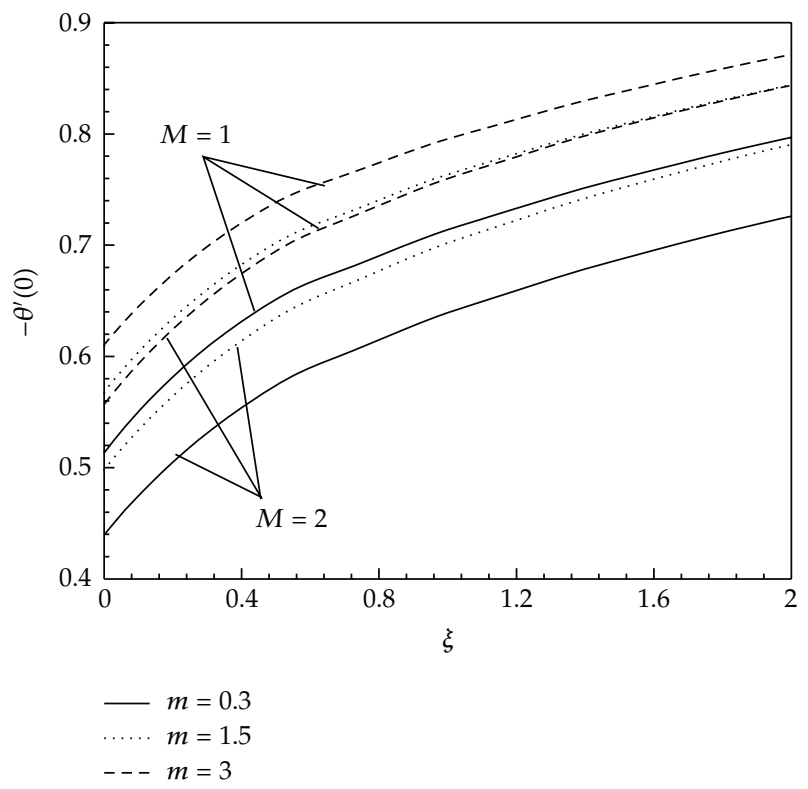

Figure 25: Local Nusselt number $-\theta^{\prime}(0)$ for various values of $M$ and $m$ for $\xi=1, R=5, a=1$, and $\operatorname{Pr}=$ 0.72 .

for fixed $m$ and $\xi$ the skin-friction coefficient in the $x$-direction $f^{\prime \prime}(0)$ and the local Nusselt number $-\theta^{\prime}(0)$ decrease while the local skin-friction coefficient in the $z$-direction $h^{\prime}(0)$ increases with increasing the magnetic parameter $M$. Further, it is remarkable to note that the effect of the magnetic parameter $M$ on $f^{\prime \prime}(0)$ and $-\theta^{\prime}(0)$ for higher values of $m$ ( $m=1.5$ and $3)$ is much less than that for lower values of $m(m=0.3)$ while the opposite trend is noticed for the local skin-friction coefficient in the $z$-direction $h^{\prime}(0)$. On the other hand, for all $\xi$ and $M$ values Figures 23 and 25 show that the local skin-friction in the $x$-direction $f^{\prime \prime}(0)$ and 
Table 1: Comparison of the values of $-\theta^{\prime}(0)$ obtained by HAM for $M=m=\xi=0$ and $R \rightarrow \infty$ and various values of Pr and $a$ with those of Magyari and Keller [25].

\begin{tabular}{lcccccccc}
\hline \multirow{2}{*}{ Pr } & \multicolumn{2}{c}{$a=-0.5$} & \multicolumn{2}{c}{$a=0.0$} & \multicolumn{2}{c}{$a=1$} & \multicolumn{2}{c}{$a=3$} \\
& Reference [25] & HAM & Reference [25] & HAM & Reference [25] & HAM & Reference [25] & HAM \\
\hline 0.5 & 0.175815 & 0.174455 & 0.330493 & 0.330824 & 0.594338 & 0.594112 & 1.008405 & 1.007963 \\
1 & 0.299876 & 0.299795 & 0.549643 & 0.549589 & 0.954782 & 0.955325 & 1.560294 & 1.560759 \\
3 & 0.634113 & 0.635700 & 1.122188 & 1.123344 & 1.869075 & 1.869397 & 2.938535 & 2.934606 \\
5 & 0.870431 & 0.874551 & 1.521243 & 1.524815 & 2.500135 & 2.500382 & 3.886555 & 3.889363 \\
10 & 1.308613 & 1.309138 & 2.257429 & 2.256776 & 3.660379 & 3.673949 & 5.628198 & 5.625053 \\
\hline
\end{tabular}

the local Nusselt number $-\theta^{\prime}(0)$ increase as $m$ increases. Further, from Figure 24 it is interesting to note that for all values of $\xi$ and $M$ the local skin friction coefficient in the $z$-direction $h^{\prime}(0)$ increases as $m$ increases from 0.3 to 1.5 and then decreases as $m$ increases from 1.5 to 3 and this result agrees well with the profiles of $h(\eta)$ introduced in Figure 9.

Finally, Table 1 shows a good agreement of our results for heat transfer rate $-\theta^{\prime}(0)$ obtained by homotopy analysis method with the numerical results reported by Magyari and Keller [25] for forced convective flow $(\xi=0)$ of a viscous incompressible fluid over an exponentially stretching sheet in the absence of magnetic field $(M=0)$, Hall current $(m=0)$, and radiation $(R \rightarrow \infty)$ at selected values of $a$ and Pr.

\section{Acknowledgments}

This research is supported by King Khalid University (KKU), Saudi Arabia, under the project no. KKU-SCI-11-10. This support is highly appreciated and acknowledged. The authors are very thankful to the reviewers for their encouraging comments and constructive suggestions to improve the presentation of this work.

\section{References}

[1] B. C. Sakiadis, "Boundary layer behavior on continuous solid surface, the boundary layer on a continuous flat surface," AIChE Journal, vol. 7, pp. 221-225, 1961.

[2] L. J. Crane, "Flow past a stretching plate," Journal of Applied Mathematics and Physics, vol. 21, no. 4, pp. 645-647, 1970.

[3] F. K. Tsou, E. M. Sparrow, and R. J. Goldstein, "Flow and heat transfer in the boundary layer on a continuous moving surface," International Journal of Heat and Mass Transfer, vol. 10, no. 2, pp. 219-235, 1967.

[4] L. J. Grubka and K. M. Bobba, "Heat transfer characteristics of a continuous, stretching surface with variable temperature," Journal of Heat Transfer, vol. 107, no. 1, pp. 248-250, 1985.

[5] L. E. Erickson, L. T. Fan, and V. G. Fox, "Heat and mass transfer on a moving continuous flat plate with suction or injection," Industrial and Engineering Chemistry Fundamentals, vol. 5, no. 1, pp. 19-25, 1966.

[6] E. M. Abo-Eldahab and M. A. El Aziz, "Blowing/suction effect on hydromagnetic heat transfer by mixed convection from an inclined continuously stretching surface with internal heat generation/absorption," International Journal of Thermal Sciences, vol. 43, no. 7, pp. 709-719, 2004.

[7] R. Cortell, "Flow and heat transfer of a fluid through a porous medium over a stretching surface with internal heat generation/absorption and suction/blowing," Fluid Dynamics Research, vol. 37, no. 4, pp. 231-245, 2005.

[8] I. C. Liu, "Flow and heat transfer of an electrically conducting fluid of second grade in a porous medium over a stretching sheet subject to a transverse magnetic field," International Journal of NonLinear Mechanics, vol. 40, no. 4, pp. 465-474, 2005. 
[9] A. Ali and A. Mehmood, "Homotopy analysis of unsteady boundary layer flow adjacent to permeable stretching surface in a porous medium," Communications in Nonlinear Science and Numerical Simulation, vol. 13, no. 2, pp. 340-349, 2008.

[10] T. S. Chen and F. A. Strobel, "Buoyancy effects in boundary layer adjacent to a continuous, moving horizontal flat plate," Journal of Heat Transfer, vol. 102, no. 1, pp. 170-172, 1980.

[11] J. R. Fan, J. M. Shi, and X. Z. Xu, "Similarity solution of mixed convection over a horizontal moving plate," Heat and Mass Transfer, vol. 32, no. 3, pp. 199-206, 1997.

[12] C. H. Chen, "Laminar mixed convection adjacent to vertical, continuously stretching sheets," Heat and Mass Transfer, vol. 33, no. 5-6, pp. 471-476, 1998.

[13] M. Ali and F. Al-Yousef, "Laminar mixed convection from a continuously moving vertical surface with suction or injection," Heat and Mass Transfer, vol. 33, no. 4, pp. 301-306, 1998.

[14] M. Ali and F. Al-Yousef, "Laminar mixed convection boundary layers induced by a linearly stretching permeable surface," International Journal of Heat and Mass Transfer, vol. 45, no. 21, pp. 4241-4250, 2002.

[15] M. A. El-Aziz and A. M. Salem, "MHD-mixed convection and mass transfer from a vertical stretching sheet with diffusion of chemically reactive species and space or temperature dependent heat source," Canadian Journal of Physics, vol. 85, no. 4, pp. 359-373, 2007.

[16] A. Moutsoglou and T.S. Chen, "Buoyancy effects in boundary layers on inclined, continuous, moving sheets," Journal of Heat Transfer, vol. 102, no. 2, pp. 371-373, 1980.

[17] F. A. Strobel and T. S. Chen, "Buoyancy effects on heat and mass transfer in boundary layers adjacent to inclined, continuous, moving sheets," Numerical Heat Transfer, vol. 3, no. 4, pp. 461-481, 1980.

[18] C. H. Chen, "Mixed convection cooling of a heated, continuously stretching surface," Heat Mass Transfer, vol. 36, no. 1, pp. 79-86, 2000.

[19] W. G. England and A. F. Emery, "Thermal radiation effects on laminar free convection boundary layer of an absorbing gas," Journal of Heat Transfer, vol. 31, pp. 37-44, 1969.

[20] R. S. R. Gorla and I. Pop, "Conjugate heat transfer with radiation from a vertical circular pin in a non-newtonian ambient medium," Wärme- und Stoffübertragung, vol. 28, no. 1-2, pp. 11-15, 1993.

[21] A. Raptis, "Radiation and free convection flow through a porous medium," International Communications in Heat and Mass Transfer, vol. 25, no. 2, pp. 289-295, 1998.

[22] M. Abd-El Aziz, "Thermal radiation effects on magnetohydrodynamic mixed convection flow of a micropolar fluid past a continuously moving semi-infinite plate for high temperature differences," Acta Mechanica, vol. 187, no. 1-4, pp. 113-127, 2006.

[23] M. Abd El-Aziz, "Thermal-diffusion and diffusion-thermo effects on combined heat and mass transfer by hydromagnetic three-dimensional free convection over a permeable stretching surface with radiation," Physics Letters A, vol. 372, no. 3, pp. 263-272, 2008.

[24] M. Abd El-Aziz, "Radiation effect on the flow and heat transfer over an unsteady stretching sheet," International Communications in Heat and Mass Transfer, vol. 36, no. 5, pp. 521-524, 2009.

[25] E. Magyari and B. Keller, "Heat and mass transfer in the boundary layers on an exponentially stretching continuous surface," Journal of Physics D, vol. 32, no. 5, pp. 577-585, 1999.

[26] E. M. A. Elbashbeshy, "Heat transfer over an exponentially stretching continuous surface with suction," Archives of Mechanics, vol. 53, no. 6, pp. 643-651, 2001.

[27] S. K. Khan and E. Sanjayanand, "Viscoelastic boundary layer flow and heat transfer over an exponential stretching sheet," International Journal of Heat and Mass Transfer, vol. 48, no. 8, pp. 15341542, 2005.

[28] E. Sanjayanand and S. K. Khan, "On heat and mass transfer in a viscoelastic boundary layer flow over an exponentially stretching sheet," International Journal of Thermal Sciences, vol. 45, no. 8, pp. 819-828, 2006.

[29] M. K. Partha, P. V. S. N. Murthy, and G. P. Rajasekhar, "Effect of viscous dissipation on the mixed convection heat transfer from an exponentially stretching surface," Heat and Mass Transfer, vol. 41, no. 4, pp. 360-366, 2005.

[30] M. A. El-Aziz, "Viscous dissipation effect on mixed convection flow of a micropolar fluid over an exponentially stretching sheet," Canadian Journal of Physics, vol. 87, no. 4, pp. 359-368, 2009.

[31] G. W. Sutton and A. Sherman, Engineering Magnetohydrodynamics, McGraw-Hill, New York, NY, USA, 1965.

[32] I. Pop and T. Watanabe, "Hall effects on magnetohydrodynamic free convection about a semi-infinite vertical flat plate," International Journal of Engineering Science, vol. 32, no. 12, pp. 1903-1911, 1994.

[33] E. M. Abo-Eldahab and M. A. El Aziz, "Hall and ion-slip effects on MHD free convective heat generating flow past a semi-infinite vertical flat plate," Physica Scripta, vol. 61, no. 3, pp. 344-348, 2000 . 
[34] A. A. Megahed, S. R. Komy, and A. A. Afify, "Similarity analysis in magnetohydrodynamics: hall effects on free convection flow and mass transfer past a semi-infinite vertical flat plate," International Journal of Non-Linear Mechanics, vol. 38, no. 4, pp. 513-520, 2003.

[35] E. M. Abo-Eldahab and M. A. El Aziz, "Hall current and ohmic heating effects on mixed convection boundary layer flow of a micropolar fluid from a rotating cone with power-law variation in surface temperature," International Communications in Heat and Mass Transfer, vol. 31, no. 5, pp. 751-762, 2004.

[36] E. M. Abo-Eldahab and M. A. El Aziz, "Viscous dissipation and Joule heating effects on MHD-free convection from a vertical plate with power-law variation in surface temperature in the presence of Hall and ion-slip currents," Applied Mathematical Modelling, vol. 29, no. 6, pp. 579-595, 2005.

[37] L. K. Saha, M. A. Hossain, and R. S. R. Gorla, "Effect of Hall current on the MHD laminar natural convection flow from a vertical permeable flat plate with uniform surface temperature," International Journal of Thermal Sciences, vol. 46, no. 8, pp. 790-801, 2007.

[38] E. M. Abo-Eldahab, M. A. El-Aziz, A. M. Salem, and K. K. Jaber, "Hall current effect on MHD mixed convection flow from an inclined continuously stretching surface with blowing/suction and internal heat generation/absorption," Applied Mathematical Modelling, vol. 31, no. 9, pp. 1829-1846, 2007.

[39] A. M. Salem and M. Abd El-Aziz, "Effect of Hall currents and chemical reaction on hydromagnetic flow of a stretching vertical surface with internal heat generation/absorption," Applied Mathematical Modelling, vol. 32, no. 7, pp. 1236-1254, 2008.

[40] M. Abd El-Aziz, "Flow and heat transfer over an unsteady stretching surface with Hall effect," Meccanica, vol. 45, no. 1, pp. 97-109, 2010.

[41] M. Sajid and T. Hayat, "Influence of thermal radiation on the boundary layer flow due to an exponentially stretching sheet," International Communications in Heat and Mass Transfer, vol. 35, no. 3, pp. 347-356, 2008.

[42] S. J. Liao, The Proposed Homotopy Analysis Technique for the Solution of Nonlinear Problems [Ph.D. thesis], Shanghai Jiao Tong University, 1992.

[43] S. Liao, Beyond Perturbation: Introduction to the Homotopy Analysis Method, Chapman \& Hall, Boca Raton, Fla, USA, 2003.

[44] P. J. Hilton, An Introduction to Homotopy Theory, Cambridge University Press, 1953.

[45] T. F. C. Chan and H. B. Keller, "Arc-length continuation and multigrid techniques for nonlinear elliptic eigenvalue problems," SIAM Journal on Scientific and Statistical Computing, vol. 3, no. 2, pp. 173-194, 1982.

[46] E. E. Grigolyuk and V. I. Shalashilin, The Continuation Method Applied to Nonlinear Problems in Solid Mechanics, Kluwer, 1991.

[47] G. W. Sutton and A. Sherman, Engineering Magnetohydrodynamics, McGraw-Hill, New York, NY, USA, 1965.

[48] T. G. Cowling, Magnetohydrodynamics, Interscience, New York, NY, USA, 1957.

[49] M. Kumari and G. Nath, "Unsteady MHD mixed convection flow over an impulsively stretched permeable vertical surface in a quiescent fluid," International Journal of Non-Linear Mechanics, vol. 45, no. 3, pp. 310-319, 2010.

[50] A. R. Ghotbi, H. Bararnia, G. Domairry, and A. Barari, "Investigation of a powerful analytical method into natural convection boundary layer flow," Communications in Nonlinear Science and Numerical Simulation, vol. 14, no. 5, pp. 2222-2228, 2009.

[51] Z. Ziabakhsh, G. Domairry, M. Mozaffari, and M. Mahbobifar, “Analytical solution of heat transfer over an unsteady stretching permeable surface with prescribed wall temperature," Journal of the Taiwan Institute of Chemical Engineers, vol. 41, no. 2, pp. 169-177, 2010.

[52] S. Liao, "An optimal homotopy-analysis approach for strongly nonlinear differential equations," Communications in Nonlinear Science and Numerical Simulation, vol. 15, no. 8, pp. 2003-2016, 2010. 


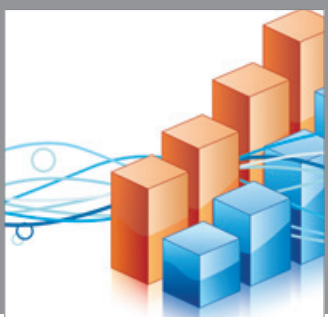

Advances in

Operations Research

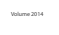

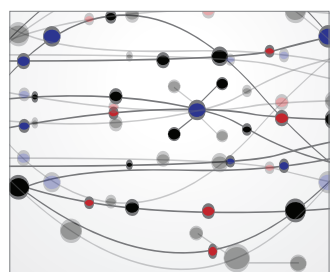

\section{The Scientific} World Journal
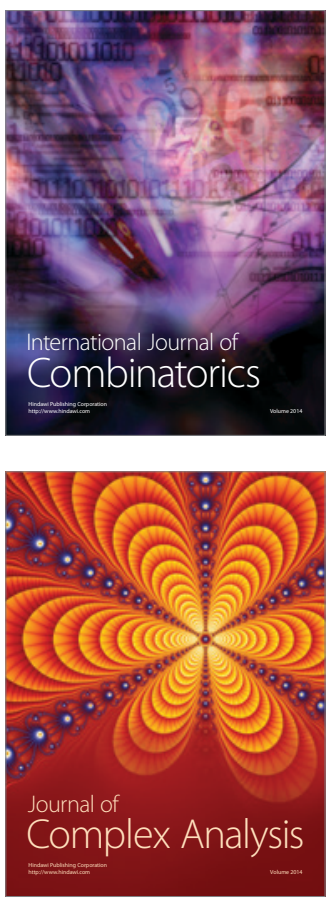

International Journal of

Mathematics and

Mathematical

Sciences
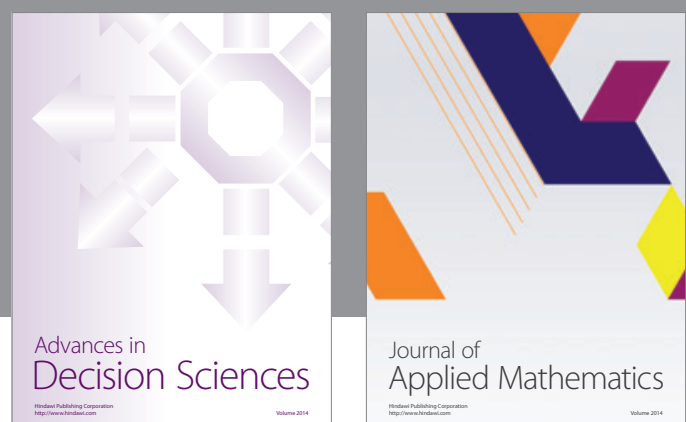

Journal of

Applied Mathematics
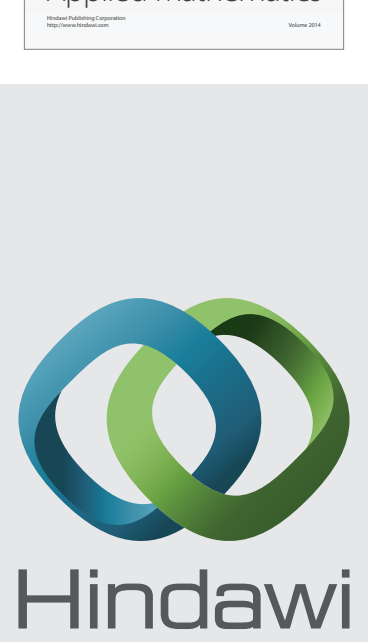

Submit your manuscripts at http://www.hindawi.com
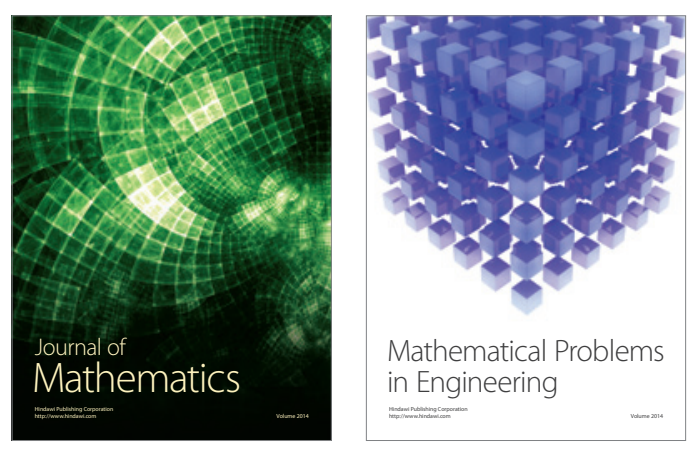

Mathematical Problems in Engineering
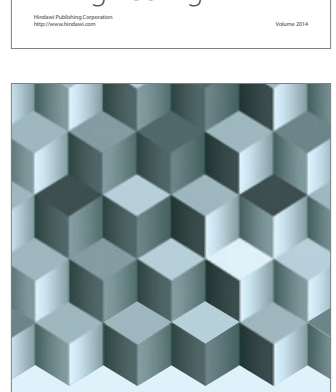

Journal of

Function Spaces
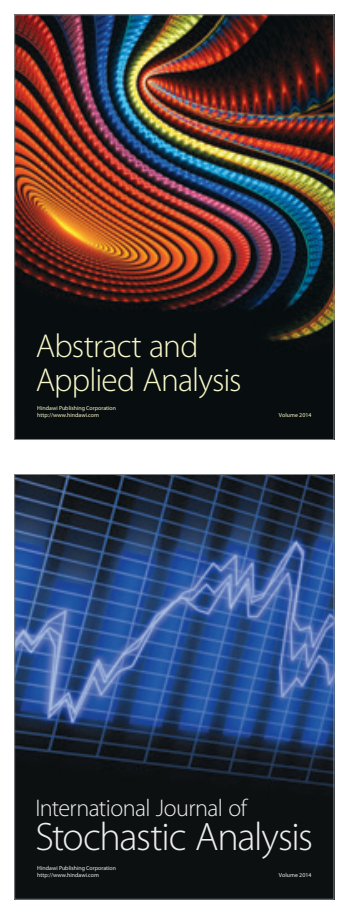

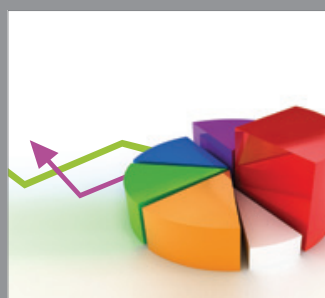

ournal of

Probability and Statistics

Promensencen
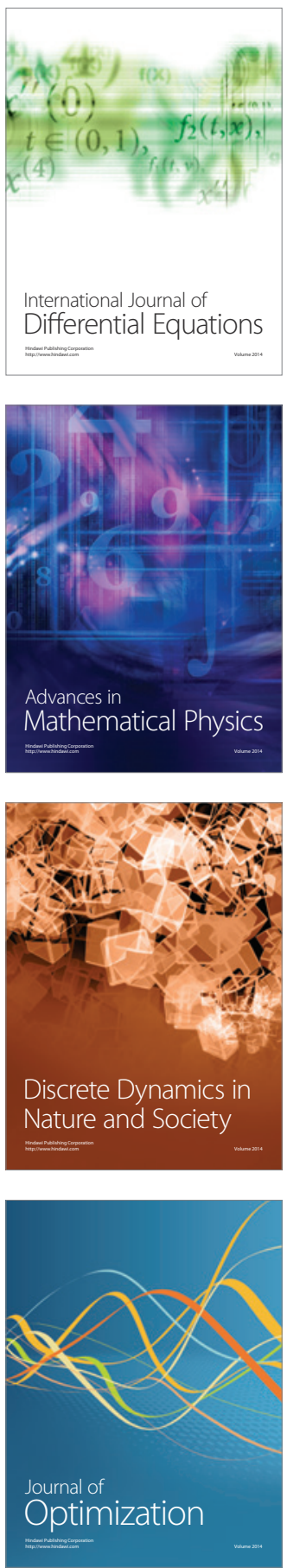